\title{
Laue's Theorem Revisited: Energy-Momentum Tensors, Symmetries, and the Habitat of Globally Conserved Quantities
}

\author{
Domenico Giulini \\ Institute for Theoretical Physics \\ Riemann Center for Geometry and Physics \\ Leibniz University of Hannover, Germany \\ giulini@itp.uni-hannover.de
}

The energy-momentum tensor for a particular matter component summarises its local energy-momentum distribution in terms of densities and current densities. We re-investigate under what conditions these local distributions can be integrated to meaningful global quantities. This leads us directly to a classic theorem by Max von Laue concerning integrals of components of the energy-momentum tensor, whose statement and proof we recall. In the first half of this paper we do this within the realm of Special Relativity and in the traditional mathematical language using components with respect to affine charts, thereby focusing on the intended physical content and interpretation. In the second half we show how to do all this in a proper differential-geometric fashion and on arbitrary space-time manifolds, this time focusing on the group-theoretic and geometric hypotheses underlying these results. Based on this we give a proper geometric statement and proof of Laue's theorem, which is shown to generalise from Minkowski space (which has the maximal number of isometries) to space-times with significantly less symmetries. This result, which seems to be new, not only generalises but also clarifies the geometric content and hypotheses of Laue's theorem. A series of three appendices lists our conventions and notation and summarises some of the conceptual and mathematical background needed in the main text.

Keywords: energy-momentum tensor, momentum map, Laue's theorem

Mathematics Subject Classification (MSC): 53D20, 58D19, 83A05

Physics and Astronomy Classification Scheme(PACS): 03.30.+p, 11.30.Cp, 11.30.-j

Acknowledgements: I thank the Collaborative Research Centre (SFB) 1227 DQ-mat of the DFG at Leibniz University of Hannover for support. 


\section{Contents}

1. The traditional picture 4

1.1. Energy-momentum tensors . . . . . . . . . . . . . . . . . 4

1.2. Global energy momentum in $\mathrm{SR} \ldots \ldots \ldots \ldots$. . . . . . . . 6

1.3. Local energy-momentum transformation . . . . . . . . . . . . . . . . 7

1.4. Global energy-momentum transformation . . . . . . . . . . . . . . 9 9

1.5. Laue's theorem: classical statement . . . . . . . . . . . . . . . . . 10

1.6. The many uses of Laue's theorem . . . . . . . . . . . . . . . . . 13

2. Geometric theory 16

2.1. Alternative representation of energy-momentum distributions . . . . . . . . . . 17

2.2. On the notions of "charges" and "conservation" . . . . . . . . . . . . . 18

2.3. Group actions and the habitat of charges . . . . . . . . . . . . . . . 19

2.4. Poincaré covariant momenta . . . . . . . . . . . . . . . . . 24

2.5. Laue's theorem: geometric formulation . . . . . . . . . . . . . 26

2.6. Recovery of the classical statement of Laue's theorem . . . . . . . . . . . . . . 27

$\begin{array}{ll}\text { 3. Conclusions and Outlook } & 29\end{array}$

$\begin{array}{ll}\text { Appendices } & \mathbf{3 0}\end{array}$

$\begin{array}{ll}\text { A. Minkowski space and Poincaré group } & \mathbf{3 0}\end{array}$

A.1. Affine spaces . . . . . . . . . . . . . . . . . . . 30

A.2. Affine automorphisms and affine groups . . . . . . . . . . . . 31

A.3. Affine bases and affine charts . . . . . . . . . . . . . . . . . 32

A.4. Passive versus active transformations . . . . . . . . . . . . . . 32

A.5. Poincaré group as metric preserving affine group . . . . . . . . . . . . 33

B. Spacetime: General conventions and notation 33

B.1. The notion of spacetime . . . . . . . . . . . . . . . 33

B.2. Some obvious bundles over manifolds . . . . . . . . . . . . . . . 33

B.3. Natural bundles . . . . . . . . . . . . . . . . . . . . . . . . . . 34

B.4. Lorentzian manifolds . . . . . . . . . . . . . . . . . . . 35

C. Definitions and convention in multilinear algebra 35

C.1. Vector spaces and the exterior algebra of its dual space . . . . . . . . . . 35

C.2. Inner products on vector spaces and their extension to the dual space and its exterior algebra . . . . . . . . . . . . . . . . . 37

C.3. Hodge duality . . . . . . . . . . . . . . . . . . 38 


\section{Introduction}

This paper deals with a well known problem in classical relativistic field-theory concerning the question of precisely how a local distribution of energy and momentum can be used to construct global quantities that, with some justification, can be said to represent these quantities globally.

The main body of the paper is organised into two Sections of approximately the same length and six subsections each. In addition there are three fairly detailed appendices providing some standard background material that we thought should not clutter the main text but the content of which is nevertheless important in order to follow the conceptual and technical arguments of the two main sections.

In Section 1 we will review and critically discuss the necessary and sufficient conditions under which meaningful global quantities may be constructed for Poincaré invariant field theories, that is, in the context of Special Relativity (henceforth abbreviated SR). We will put particular emphasis on the requirement that the global quantities in question must change according to a particular representation of the Poincaré group (inhomogeneous Lorentz group) under the action of that group on all those fields whose energy-momentum distribution is considered. This is a non-trivial requirement, which is conceptually essential for the proper physical interpretation of the quantities to be constructed. We will find that it takes the simple mathematical form of a condition of equivariance, provided a careful distinction is made between the dynamical fields (carrying energy and momentum) and auxiliary ones. This will then naturally lead us to a classic theorem dating back to 1911 due to Max von Laue (still without the nobiliary particle "von" in his name at the time he wrote the relevant paper, hence it is usually referred to simply as "Laue's theorem"), the proof and physical relevance of which we will also discuss.

The mathematical language of Section 1 deliberately sticks to the traditional component representation of geometric objects over Minkowski space. It is this component language that is most familiar to working physicists and that is often the most direct way to import the physical intuition and intentions behind various constructions. Most of what is presented in Section 1 is well known, in one form or another, though I believe that some of the subtle points are here spelled out more clearly and comprehensibly than in other sources.

In Section 2 we will then transform the preceding, coordinate-based discussion into a proper differential-geometric language. As is widely appreciated, coordinate-based language not only tends to hide the geometric assumptions that are necessary in order to render the whole construction meaningful, but it also bears the danger to mislead to geometrically meaningless constructions, like, e.g., taking integrals over tensor components - a construction often encountered in physics. This is precisely what is done in the formulation and proof of Laue's theorem, of which no proof in component-free form has hitherto been given, nor have its geometric hypotheses been spelled out correctly. We will fill that gap in Section 2 in which we give a proper statement and proof of a generalisation of Laue's theorem that remains valid outside SR and wich contains the classical statement as a special case.

Throughout, and in particular in Section 2, we shall make free use of standard differential geometric concepts, like manifolds, tensor bundles and their sections, natural bundles, various 
forms of derivatives (exterior, Lie, covariant, exterior-covariant), Hodge duality, etc. All these should be familiar to physicists used to geometric field theory, particularly those familiar with General Relativity (henceforth abbreviated by GR) on the level of a good modern text-book, like [25]. Our notation and conventions relating to these concepts are explained in detail in Appendices B and C. Appendix A contains some background material relating to Minkowski space and its automorphism group, the Poincaré group. Appendix A is also meant to remind the reader on the notion of affine spaces and associated structural elements, like affine bases, affine charts, and affine groups, as well as the distinction between active and passive transformations. Albeit basic, these notions often tend to be suppressed or even forgotten in the physics literature. Being aware of them is important in order to fully appreciate the discussion in Section 1, to which we now turn.

\section{The traditional picture}

In this section, which contains six subsections, we start with our first half of the program as just outlined. As already stressed, we deliberately adopt a traditional mathematical language, usually employed in physics papers and physics textbooks, to keep as close as possible contact to the intentions and intuitions coming from physics. At the same time we try to carefully avoid some of the misconceptions which are often imported by either assuming some unwarranted mathematical properties, or by misinterpreting them. A typical example of the latter sort results from an insufficient distinction between the two meanings of Poincaré transformations (compare Appendix A.4): First, as mere transformations between affine charts on Minkowski space $M$ (passive interpretation), which necessarily affect the component representation of all geometric structures defined over $M$, and, second, automorphism of Minkowski space (i.e. special affine maps), which we can use to act selectively on only some geometric structures which are physically distinguished in a contextual fashion. For example, we may wish to act with a boost transformation on that subset of fields in Minkowski space whose energy-momentum distribution we consider, while leaving all other fields in their original state. That is, we may wish to boost some of the matter fields relative to all the others providing the reference. This will be the situation we actually encounter below.

\subsection{Energy-momentum tensors}

The energy-momentum distribution of a physical system is characterised by its energy-momentum tensor $\mathbf{T}$, which is a section in the bundle $\mathrm{TM} \otimes \mathrm{TM}$. Following standard conventions we write $\mathbf{T} \in \Gamma(\mathrm{TM} \otimes \mathrm{TM})($ compare Appendix B). So far $(M, g)$ may represent any spacetime. Here we are restricting attention to four-dimensional $M$ (this will be relaxed in the second section). Following standard conventions in physics we denote space-time indices by greek letters if they refer to four dimensions (otherwiswe latin) and let $\alpha, \beta, . ., \in\{0,1,2,3\}$, where the index 0 refers to a timelike basis element or coordinate.

Consider a point $p \in M$ and a timelike future-pointing vector $u \in T_{p} M$ normalised to $c$ (velocity of light), so that $g(u, u)=c^{2}$. That vector defines an instantaneous state of motion of 
an observer at $p$ whose four-velocity is just $u$. We take $e_{0}:=u / c$ as a first (timelike) vector of a orthonormal basis $\left\{e_{0}, e_{1}, e_{2}, e_{3}\right\}$ of $T_{p} M$, such that $g\left(e_{\alpha}, e_{\beta}\right)=g_{\alpha \beta}=\operatorname{diag}(1,-1,-1,-1)$. We call $u_{\perp}:=\left\{v \in \mathrm{T}_{\mathrm{p}} M: g_{\mathrm{p}}(u, v)=0\right\}=\operatorname{span}\left\{e_{1}, e_{2}, e_{3}\right\}$ the instantaneous rest-space of the observer $u$.

If $M$ is Minkowski space (an affine space; see Appendix A) we may identify each $T_{p} M$ with the underlying vector space $V$. Then the subset $p+u_{\perp}$ of $M$ is a spacelike affine hyperplane consisting of all events in $M$ which are Einstein synchronous with $p$ for an inertial (i.e. force free) observer moving along the timelike line tangent to $u$ through $p$. All this is standard terminology in physics and mathematical relativity; see, e.g., [23] for a good account.

Let $\left\{\theta^{0}, \theta^{1}, \theta^{2}, \theta^{3}\right\}$ be the dual basis to $\left\{e_{0}, e_{1}, e_{2}, e_{3}\right\}$, i.e. $\theta^{\alpha}\left(e_{\beta}\right)=\delta_{\beta}^{\alpha}$. Then $T^{\alpha \beta}=$ $\mathbf{T}\left(\theta^{\alpha}, \theta^{\beta}\right)$ are the so-called contravariant components of $\mathbf{T}$, whose physical interpretation is as follows: $\mathrm{T}^{00}$ is the energy density, $\vec{S}:=\left(\mathrm{cT}^{01}, \mathrm{cT}^{02}, \mathrm{cT}^{03}\right)$ are the three components of the energy current-density, $\vec{G}:=\left(T^{10} / c, T^{20} / c, T^{30} / c\right)$ are the three components of the momentum density, and finally the symmetric $3 \times 3$ matrix $\left\{\mathrm{T}^{\mathrm{ab}}\right\}$ comprises the 9 components of the momentum current-density. Here the word "components" always refers to vectors and tensors in the instantaneous 3-dimensional rest frame $u_{\perp}$ with respect to the basis $\operatorname{span}\left\{e_{1}, e_{2}, e_{3}\right\}$. As such T comprises $1+3+3+9=16$ independent components characterising the energy-momentum distribution of matter at each spacetime point.

Often, though not always, $\mathbf{T}$ is assumed to be symmetric,

$$
\mathrm{T}^{\alpha \beta}=\mathrm{T}^{\beta \alpha} \text {. }
$$

In the notation above this means that the energy current-density $\vec{S}$ and the momentum density $\vec{G}$ are related by $\overrightarrow{\mathrm{G}}=\overrightarrow{\mathrm{S}} / \mathrm{c}^{2}$ and that the $\mathrm{n}$-th component of the $\mathrm{m}$-th momentum current-density equals the $\mathrm{m}$-th component of the $\mathrm{n}$-th momentum current-density, i.e. $\Sigma^{\mathrm{mn}}=\Sigma^{\mathrm{nm}}$. Hence a symmetry (1) reduces the 16 to $1+3+6=10$ independent components. Energy momentum tensors representing all the sources of gravitational fields in Einstein's field equations of GR are necessarily symmetric, though this does not necessarily imply that they cannot be meaningfully decomposed into a sum of non symmetric ones attributed to various sub-components of matter. In any case, we shall assume $\mathbf{T}$ to be symmetric throughout this paper.

There is another condition that $\mathbf{T}$ may satisfy and which we wish to mention here, although we shall not need it right now (it becomes of central importance later on). It is the covariant divergencelessness of $\mathbf{T}$, which we write as $\nabla \cdot \mathbf{T}=0$, or in components

$$
\nabla_{\beta} T^{\alpha \beta}=0 \text {. }
$$

Here $\nabla$ denotes the Levi-Civita covariant derivative for $\mathrm{g}$, that is, the unique torsion-free and metric (i.e $\nabla \mathrm{g}=0$ ) connection (we use "covariant derivative" and "connection" synonymously). Equation (2) implies the local conservation of energy-momentum encoded by $\mathbf{T}$, provided $\mathrm{g}$ admits certain symmetries generated by Killing vector fields. Without such symmetries energy and momentum of the matter alone will not be conserved. In that case the interpretation of (2) in GR is that locally energy and momentum can be exchanged between the matter (described by T) and the gravitational field (described by g); see, e.g., [25]. 


\subsection{Global energy momentum in SR}

Let us now restrict $(M, g)$ to Minkowski space. In manifold terms this means that $M$ is diffeomorphic to $\mathbb{R}^{4}$ and that $g=\eta$, where here and in the sequel $\eta$ denotes a Lorentz metric whose Levi-Civita connection is flat. We also recall the affine structure of Minkowski space and refer to Appendix A for details. Important here is that the affine structure endows the manifold $M$ with a preferred set of global charts, called affine charts. With respect to affine charts all connection coefficients vanish and (2) assumes the form

$$
\partial_{\beta} T^{\alpha \beta}=0,
$$

which can indeed be interpreted as local energy-momentum conservation.

Let us digress for a moment in order to make a few comment concerning the physical significance of the affine structure of Minkowski space. As is well known, the timelike lines within the set of all lines (one-dimensional affine subspaces) already fix the entire affine structure of Minkowski space. In this fashion the affine structure of Minkowski space becomes a faithful mathematical representation of the physical law of inertia, according to which a particular subset of motions is characterised as "force-free" or, as already said, "inertial". In this way affine maps, too, acquire the physical significance as the symmetry group of the law of inertia: they map force-free motions to force-free motions. In that sense the law of inertia should be regarded as a special case of a "path structure" in the sense of [5], without the specification of which the very notion of "force" makes no sense. By definition, "forces" are the causes for deviations from inertial motions, which must be defined first. For more on this, see [10] and [19]. The take-home message for us at this point is that in using affine structures we are, in fact, already in the process of modelling physical dynamical principles.

After this small digression we return to our main discussion. We note that Hypersurfaces of constant value for a single affine-chart coordinates are affine hyperplanes. In such charts a moment in time is represented by a spacelike affine hyperplane $\Sigma \subset M$ in Minkowski space; e.g., the hyperplane of constant time zero: $\Sigma:=\left\{p \in M: x^{0}(p)=0\right\}$. The global quantity we wish to construct is either associated with the whole hyperplane (i.e. involves all of space at this time) or a bounded subset thereof, which we shall also denote by $\Sigma$. For its construction we do not wish to impose (3) in order to be able to speak of the system's overall energy and momentum in situations where it is not conserved. Hence, in what follows next, we shall only assume symmetry (1). Later we will have plenty of opportunity to discuss the impact of (3)

We wish to speak of the total energy and momentum contained in $\Sigma$ (i.e. "at the moment in time" represented by $\Sigma$ ). The most naive thing to do is to just integrate the energy density $\mathrm{T}^{00}$ and momentum density ${ }^{1} \mathrm{~T}^{\mathrm{a} 0}(\mathrm{a}=1,2,3)$ over $\Sigma$. Hence we consider the four numbers, $\left\{\mathrm{P}^{\alpha}: \alpha=0,1,2,3\right\}$ defined by

$$
P^{\alpha}:=\int_{\Sigma} T^{\alpha \beta} n_{\beta} d \mu_{\Sigma}
$$

\footnotetext{
Actually, as explained above, the momentum density is $1 / c$ times the components $T^{a 0}(a=1,2,3)$, which means that our $\mathrm{P}^{\alpha}$ will actually equal $c$ times the components of ordinary four-momentum. In order not to carry along all the factors of $c$ we shall ignore this difference, which is completely irrelevant for our purpose and which disappears in units where $c=1$.
} 
Here $n_{\beta}$ are the covariant components of the normal to $\Sigma$ in $M$ and $d \mu_{\Sigma}$ is the measure 3-form on $\Sigma$ induced from the measure 4-form $\varepsilon$ on $(M, g)$, which in affine coordinates $x^{\alpha}$ is just the Lebesgue measure: $\varepsilon=d x^{0} \wedge d x^{1} \wedge d x^{2} \wedge d x^{3}$. Writing $i_{n}$ for the insertion map that inserts $n$ into the first slot of a general form, we have

$$
\mathrm{d} \mu_{\Sigma}=\star \mathrm{n}^{b}=i_{n} \varepsilon=\frac{1}{3 !} \mathrm{n}^{\mu} \varepsilon_{\mu \alpha \beta \gamma} \mathrm{d} x^{\alpha} \wedge d x^{\beta} \wedge d x^{\gamma} .
$$

Here and in the following the superscript $b$ on a vector denotes its corresponding dual vector under the isomorphism given by the metric, which we define in (90). Also $\star$ denotes the Hodge duality map defined in (98).

The intended result of the somewhat bold method to just integrate all the densities is, of course, that these four numbers somehow represent the total energy and total translatory momentum of the system in the rest-frame represented by the hyperplane $\Sigma$ and with respect to the affine coordinates $\chi^{a}$ used within in. We further expect these four numbers to define an element in a vector space that carries a specific representation of the Lorentz group, namely the defining one. Physicists often express this expectation by saying that total energy-momentum should form a "four vector", meaning that the four numbers transform according to the defining representation of $\mathrm{SO}(1,3)$. But is that really true? And if so, how would we characterise the vector space this "energy-momentum vector" is an element of? In fact, as is well known, and as we will see in a moment, the fulfilment of all these expectations is not at all guaranteed and depends on further global conditions on $\mathbf{T}$ that we will now investigate.

\subsection{Local energy-momentum transformation}

The four numbers (4) we now calculate for two different dynamical systems whose energymomentum distributions are represented by two different energy-momentum tensors, $\mathbf{T}$ and $\overline{\mathbf{T}}$. The two systems are chosen such that they are related by an active Lorentz transformation $\Lambda$, which means that

$$
(\Lambda, \mathbf{T}) \mapsto \Lambda \cdot \mathbf{T}:=\Lambda \otimes \Lambda\left(\mathbf{T} \circ \Lambda^{-1}\right)=: \overline{\mathbf{T}}
$$

where in the last step we simply introduced the notational abbreviation of writing an overbar for the $\Lambda$-transformed quantity. Note that (6) just denotes the push-forward transformation law of a contravariant tensor field under the action of the Lorentz transformation $\Lambda$. It is a special example for the natural lift of the diffeomorphic action of the Lorentz group on $M$ to any bundle associated to the principal bundle of linear frames, here to the bundle $T M \otimes T M$ (compare Appendix B.3). In terms of component functions with respect to our affine coordinates, this reads

$$
\overline{\mathrm{T}}^{\alpha \beta}(x)=\Lambda_{\gamma}^{\alpha} \Lambda_{\delta}^{\beta} T^{\gamma \delta}\left(\Lambda^{-1} x\right),
$$

where the $x$ and $\Lambda^{-1} x$ in the argument stands for all four $x^{\mu}$ and $\left(\Lambda^{-1}\right)_{\nu}^{\mu} x^{\nu}, \mu \in\{0,1,2,3\}$, respectively.

The following is important to note: For both dynamical systems the components of their respective dynamical quantities refer to the same affine coordinates $\left\{x^{\alpha}: \alpha=0,1,2,3\right\}$. Our Poincaré transformation acts on the dynamical fields, not on the observer or a reference-system, 
and produces via its action another system with different observable features relative to the very same reference system that we exclusively use.

To end this subsection we wish to alert the reader to another non-trivial aspect hidden in (6), also connection with the active interpretation of transformations employed here, which is the following: Usually we think of $\mathbf{T}$ not as just simply given, but as functionally specified in terms of elementary fields, which we separate into two sets, $\mathrm{F}$ and $\mathrm{F}^{\prime}$, whose distinction is important. Hence we write $\mathbf{T}\left(F, F^{\prime}\right)$. $F$ collectively denotes all fields of which $\mathbf{T}$ represents the energymomentum distribution, and $F^{\prime}$ collectively denotes all possibly existent further background fields that we may use to define T. For example, in Maxwellian electrodynamics in its traditional "metric" formulation ${ }^{2}, \mathrm{~F}$ would be the Faraday tensor, comprising electric and magnetic fields, and $F^{\prime}$ would be the background Minkowskian metric. The value-space of the dynamical fields $\mathrm{F}$ is assumed to carry some representation $\mathrm{D}$ of the Lorentz group, so that the field $\mathrm{F}$ itself (as element of a mapping space; later to be identified with a section in an appropriate bundle) transforms as

$$
(\Lambda, F) \mapsto \Lambda \cdot F:=D(\Lambda) \circ F \circ \Lambda^{-1} .
$$

The non-trivial requirement behind (6) then is:

$$
\mathbf{T}\left(\Lambda \cdot F, F^{\prime}\right)=\Lambda \cdot \mathbf{T}\left(F, F^{\prime}\right) .
$$

Note that the corresponding statement

$$
\mathbf{T}\left(\Lambda \cdot F, \Lambda \cdot F^{\prime}\right)=\Lambda \cdot \mathbf{T}\left(F, F^{\prime}\right),
$$

in which $\Lambda$ is also allowed to act on $F^{\prime}$ as $\left(\Lambda, F^{\prime}\right) \mapsto \Lambda \cdot F^{\prime}:=D^{\prime}(\Lambda) \circ F^{\prime} \circ \Lambda^{-1}$ via some representation $\mathrm{D}^{\prime}$ on the value-space of $\mathrm{F}^{\prime}$, would be much weaker and, in fact, almost trivial. It would merely require $\mathbf{T}$ to be a covariant construct of $F$ and $F^{\prime}$. In contrast, equation (9) is far more demanding. It requires that the energy-momentum distribution of the $\Lambda$-shifted fields $F$ is the $\Lambda$-shift of the original energy-momentum distribution of $F$, given the same background $\mathrm{F}^{\prime}$ throughout. For example, coming back to ordinary (metric) electromagnetism in Minkowski space, let now $\Lambda$ be any diffeomorphism, $F$ again the Farady tensor, and $F^{\prime}$ the background Minkowski metric. Then (10) will always hold true, but (9) only if $\Lambda$ stabilizes $F^{\prime}$, i.e. $\Lambda \cdot F^{\prime}=F^{\prime}$, which just says that $\Lambda$ must be an isometry and hence a Poincaré transformation.

It seems remarkable that the non-trivial requirement behind (9) is hardly ever spelled out, or even recognised, in standard text-books. A notable exception is found in $\S 31$ and $\S 31^{*}$ of Fock's classic text [8], where Fock discusses the requirement (which he calls a "physical principle") that "the mass tensor must be a function of the state of the system" ([8], p. 95). Here "mass tensor" refers to the energy-momentum tensor rescaled by $c^{-1}$. This sound as if Fock had (9) in mind, which is also supported by his discussion that stresses the non-triviality of his "physical principle". However, semantically an ambiguity remains as to whether Fock's "system" refers to, in our notation, the system represented by $F$, or the wider system represented by $\left(F, F^{\prime}\right)$. Fock unfortunately does not explicitly address this distinction which becomes particularly important in the more general cases discussed below in 2.3.

\footnotetext{
2 The metric formulation of electrodynamics assumes a background metric of spacetime, the Minkowski metric. There are also metric-free formulations, as discussed in detail in [13].
} 


\subsection{Global energy-momentum transformation}

We now wish to see how the four numbers (4) are affected by a particular set of Lorentz transformations, namely pure boosts. ${ }^{3}$ We restrict to boosts because no non-trivial statement of the kind developed here follows from translations and rotations.

So let us specifically take a boost in $x^{1}$-direction:

$$
\Lambda_{\beta}^{\alpha}=\left(\begin{array}{cccc}
\gamma & \beta \gamma & 0 & 0 \\
\beta \gamma & \gamma & 0 & 0 \\
0 & 0 & 1 & 0 \\
0 & 0 & 0 & 1
\end{array}\right)
$$

Here, as usual, $\beta:=v / c$ and $\gamma:=1 / \sqrt{1-\beta^{2}}$. Then

$$
\begin{aligned}
& \overline{\mathrm{T}}^{00}(x)=\gamma^{2}\left[\mathrm{~T}^{00}(\underline{x})+\beta^{2} \mathrm{~T}^{11}(\underline{x})+2 \beta \mathrm{T}^{01}(\underline{x})\right] \\
& \overline{\mathrm{T}}^{11}(x)=\gamma^{2}\left[\mathrm{~T}^{11}(\underline{x})+\beta^{2} \mathrm{~T}^{00}(\underline{x})+2 \beta \mathrm{T}^{01}(\underline{x})\right] \\
& \overline{\mathrm{T}}^{01}(x)=\gamma^{2}\left[\left(1+\beta^{2}\right) \mathrm{T}^{01}(\underline{x})+\beta\left(\mathrm{T}^{00}(\underline{x})+\mathrm{T}^{11}(\underline{x})\right)\right] \\
& \overline{\mathrm{T}}^{0 \mathrm{n}}(x)=\gamma\left[\mathrm{T}^{0 \mathrm{n}}(\underline{x})+\beta \mathrm{T}^{1 \mathrm{n}}(\underline{x})\right] \\
& \overline{\mathrm{T}}^{1 \mathrm{n}}(x)=\gamma\left[\mathrm{T}^{1 \mathrm{n}}(\underline{x})+\beta \mathrm{T}^{0 \mathrm{n}}(\underline{x})\right] \\
& \overline{\mathrm{T}}^{\mathrm{nm}}(x)=\mathrm{T}^{\mathrm{nm}}(\underline{x}) .
\end{aligned}
$$

Here we set for abbreviation,

$$
\underline{x}:=\Lambda^{-1} x=\left(\gamma\left(x^{0}-\beta x^{1}\right), \gamma\left(x^{1}-\beta x^{0}\right), x^{2}, x^{3}\right),
$$

and the indices $n$ and $m$ may take on independently any of the two values 2 and 3 .

In the integral (4) we have $n^{\mu}=1$ for $\mu=0$ and $n^{\mu}=0$ otherwise, for $\Sigma$ is the zero-level set of $x^{0}$. This implies that the induced measure (5) is simply the Lebesgue measure in the spatial affine coordinates

$$
d \mu_{\Sigma}=d x^{1} \wedge d x^{2} \wedge d x^{3}=: d^{3} x .
$$

For $\overline{\mathrm{P}}^{\alpha}$ the integrals (4) now involve the four components $\overline{\mathrm{T}}^{00}, \overline{\mathrm{T}}^{01}$ and $\overline{\mathrm{T}}^{0 \mathrm{n}}(\mathrm{n}=2,3)$, evaluated on $\Sigma$. For $x^{0}=0$ we have $\underline{x}=\left(-\beta \gamma x^{1}, \gamma x^{1}, x^{2}, x^{3}\right)$. Therefore the integrands (4) will involve the components $\overline{\mathrm{T}}^{00}, \overline{\mathrm{T}}^{01}$ and $\overline{\mathrm{T}}^{0 \mathrm{n}}$ at all times $-\beta \gamma x^{1}$, where $x^{1}$ takes all values from within the intersection of $\Sigma$ with the support of $\mathbf{T}$. As a result, no simple statement relating the integrals of the original and the boosted systems can be made for generally time dependent $T^{\alpha \beta}$. On the other hand, if we assume the components $T^{\alpha \beta}$ to be independent of time $x^{0}$, i.e.,

$$
\partial_{0} T^{\alpha \beta}=0,
$$

\footnotetext{
3 Pure boosts can be characterised within the Lorentz group only with reference to a timelike direction, which here is taken to be $\partial / \partial x^{0}$. A 'pure boost' is then any linear Lorentz transformation that moves points in a timelike 2-plane containing $\partial / \partial x^{0}$ and leaves the g-orthogonal spacelike 2-plane poinwise fixed.Pure boosts do not form a subgroup of the Lorentz group.
} 
all dependencies of the barred components $\overline{\mathrm{T}}^{\alpha \beta}$ on the spatial coordinates derive from the spatial dependence of $T^{\alpha \beta}$ in the simple fashion given by (12g) for $\chi^{0}=0: x^{1}$ is multliplied with $\gamma$ whereas the dependence on $x^{2}$ and $x^{3}$ is unchanged. By a simple change of variables in the integration over $\Sigma$ this results in a common factor $1 / \gamma$ and we obtain

$$
\begin{aligned}
& \overline{\mathrm{P}}^{0}=\gamma\left(\mathrm{P}^{0}+2 \beta \mathrm{P}^{1}+\int_{\Sigma} \mathrm{T}^{11} \mathrm{~d}^{3} x\right) \\
& \overline{\mathrm{P}}^{1}=\gamma\left(\left(1+\beta^{2}\right) \mathrm{P}^{1}+\beta \mathrm{P}^{0}+\beta \int_{\Sigma} \mathrm{T}^{11} \mathrm{~d}^{3} x\right), \\
& \overline{\mathrm{P}}^{\mathrm{n}}=\mathrm{P}^{\mathrm{n}}+\beta \int_{\Sigma} \mathrm{T}^{1 n} \mathrm{~d}^{3} x
\end{aligned}
$$

This we compare to the defining representation of the Lorentz group on $\mathbb{R}^{4}$ :

$$
\begin{aligned}
& \bar{X}^{0}=\gamma\left(X^{0}+\beta X^{1}\right), \\
& \bar{X}^{1}=\gamma\left(X^{1}+\beta X^{0}\right), \\
& \bar{X}^{n}=X^{n} .
\end{aligned}
$$

It follows that the $\mathrm{P}^{\alpha} \rightarrow \overline{\mathrm{P}}^{\alpha}$ transformation follows the pattern (16) for all $\beta$, if and only if $\mathrm{P}^{1}=0$ and all three integrals $\int_{\Sigma} T^{1 a} d^{3} x$ vanish for $a=1,2,3$.

Now, as the direction of our boost was arbitrary, repeating the argument with boosts in the 2 - and 3-direction shows that $\mathrm{P}^{\alpha} \rightarrow \overline{\mathrm{P}}^{\alpha}$ transforms like the components of four vector under boosts, if and only if the integrals over $\Sigma$ of all components $T^{\mu \nu}$ vanish, except that of $T^{00}$. Hence

$$
\int_{\Sigma} T^{\mu m} d^{3} x=0
$$

for all $\mu \in\{0,1,2,3\}$ and all $m \in\{1,2,3\}$.

A priori there seems to be no obvious reason why any stationary physical system should have an energy-momentum tensor satisfying (17), In fact, (17) will fail in the general stationary case. But recall that so far we did not assume energy-momentum conservation (3). It is Laue's theorem that precisely connects energy-momentum conservation and (17).

\subsection{Laue's theorem: classical statement}

Laue's theorem was first proven (under slightly stronger hypotheses, some of which turn out to be unnecessary) in his classic paper [17], in which he for the first time explained in a general fashion the impact of Special Relativity onto the dynamical description of systems in static equilibrium. The theorem is based on the obvious identity,

$$
\partial_{n}\left(T^{\mu n} \varphi\right)=\left(\partial_{n} T^{\mu n}\right) \varphi+T^{\mu n} \partial_{n} \varphi,
$$

where $\varphi: \Sigma \rightarrow \mathbb{R}$ is any smooth function. If we assume energy-momentum conservation (3) and stationarity (14), the first term on the right-hand side vanishes. Applying Gauß' theorem 
(Stokes' theorem in 3-dimensions), we get

$$
\int_{\Sigma} T^{\mu n} \partial_{n} \varphi d^{3} x=\int_{\partial \Sigma} T^{\mu n} \varphi v_{n} d o
$$

where $v_{n}$ are covariant components of the outward-pointing normal of the boundary $\partial \Sigma$ of $\Sigma$ and do is the induced measure on the 2-dimensional boundary. Now, if $\left.\left(T^{\mu n} \varphi\right)\right|_{\partial \Sigma}=0$, e.g., for all $\varphi$ with compact support in the interior of $\Sigma$, we have

$$
\int_{\Sigma} T^{\mu n} \partial_{n} \varphi d^{3} x=0
$$

If we forget about the index $\mu$ for a moment, this is just the well-known statement that a divergence-free vector field is $\mathrm{L}^{2}\left(\mathbb{R}^{3}, \mathrm{~d}^{3} \mathrm{x}\right.$ )-orthogonal to all gradient fields (given sufficient falloff conditions in the case of non-compact support).

Now, for the special case $\varphi=x^{\mathfrak{m}}$ this reduces to (17), provided that the boundary integral in (19) still vanishes. This poses further conditions on $\mathbf{T}$ regarding its behaviour near $\partial \Sigma$. An obvious sufficient condition would be that the intersection of T's support

$$
\operatorname{supp}(\mathbf{T}):=\overline{\{p \in M: \mathbf{T}(p) \neq 0\}}
$$

with $\Sigma$ is compact. On the other hand, if $\operatorname{supp}(\mathbf{T}) \cap \Sigma$ is not compact, it would be sufficient that T has a $1 / r^{3+\epsilon}$ fall-off (i.e. faster than $1 / r^{3} ; \epsilon>0$ ) at each end ${ }^{4}$ of $\Sigma$, where $r$ denotes spatial geodesic distance. Note that for radiating systems $\Sigma$ needs to be a Cauchy surface in order for the requirement of compact support to make sense. The reason for this is explained in Fig. 1.

The foregoing discussion shows the following

Theorem 1 (Laue's theorem, classical version). Let $\mathbf{T}$ be a symmetric second-rank contravariant tensor in Minkowski space $(\mathrm{M}, \eta)$ - from now on called the energy-momentum tensor -, which is such that there exists a global affine chart $\left\{x^{0}, x^{1}, x^{2}, x^{3}\right\}$ such that $\partial \mathrm{T}^{\alpha \beta} / \partial x^{0}=0$. Let $\Sigma \subset M$ be the spacelike hyperplane $x^{0}=0$, or a bounded subdomain thereof, which we fix once and for all. Consider now the four intgrals (4), which we write $\mathrm{P}^{\alpha}[\Sigma, \mathbf{T}]$, and the corresponding four integrals for the Lorentz-boosted energy-momentum tensor $\Lambda \cdot \mathbf{T}:=\Lambda \otimes \Lambda\left(\mathbf{T} \circ \Lambda^{-1}\right)$. Then

$$
\mathrm{P}^{\alpha}[\Sigma, \Lambda \cdot \mathbf{T}]=\Lambda_{\beta}^{\alpha} \mathrm{P}^{\beta}[\Sigma, \mathbf{T}]
$$

for all pure boost transformations, if and only if the nine space integrals (17) vanish. A sufficient condition for this to happen is that $\mathbf{T}$ is conserved in the sense of (3) and either $\left.\mathbf{T}\right|_{\partial \Sigma} \equiv 0$ (in case $\partial \Sigma \neq \emptyset$ ) or $\mathbf{T}$ falls off like $1 / \mathrm{r}^{3+\epsilon}$ at each end of $\Sigma$, where $r$ is the geodesic distance with respect to any reference point inside $\Sigma$.

The classic literature on special relativity is full of more or less convincing derivations of this result. Laues original derivation [17] and all (as far as I am aware) of its followers, even the most

\footnotetext{
4 "Ends" of manifolds are connected subsets not contained in any compact connected subset. This notion was coined in [9]. They are sometimes called "asymptotic regions" in the physics literature.
} 


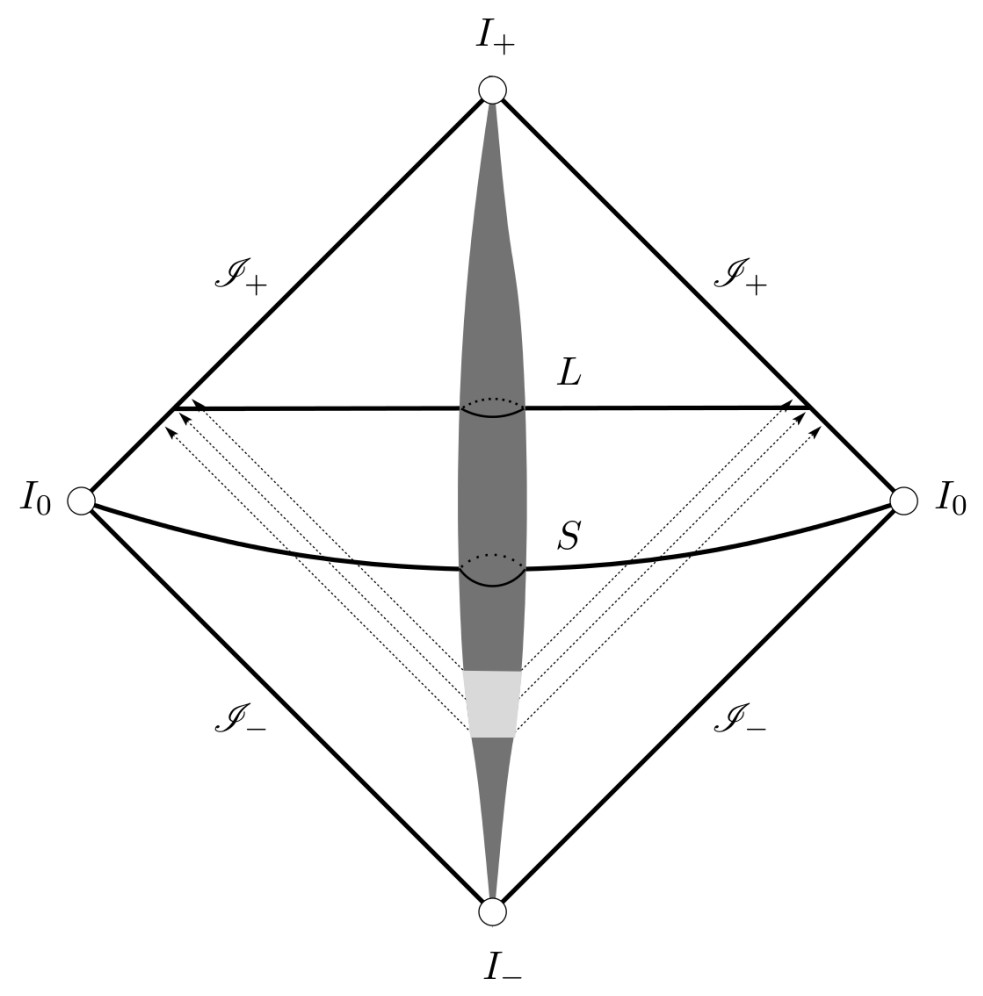

Figure 1: The figure shows the conformal compactification of Minkowski space, the boundary of which decomposes into future/past timelike infinity $\mathrm{I}_{+} / \mathrm{I}_{-}$, future/past lightlike infinity $\mathcal{J}_{+} / \mathcal{J}_{-}$, and spacelike infinity $\mathrm{I}_{0}$. The vertical dark-shaded region extending from $\mathrm{I}_{-}$to $\mathrm{I}_{+}$corresponds to the "world tube" of the physical system whose energymomentum tensor is denoted by $\mathbf{T}$ in the text. The lighter shaded region within that denotes the events during which the system radiates. The dotted straight lines at 45 degrees connecting that lighter shaded region to $\mathcal{J}_{+}$indicate the lightlike rays of radiation. We have drawn two spacelike hypersurfaces: One denoted by $S$, which is asymptotically flat and extends all the way to $\mathrm{I}_{0}$, and another one, $\mathrm{L}$, which is asyptotically hyperboloidal (of constant negative sectional curvature) and which extends to $\mathcal{J}_{+}$. The former is a Cauchy hypersurface, i.e. its domain of dependence is all of Minkowski space, the other is not. For the latter it is clear that the radiation that the system released during the finite time interval (light shaded region) in the finite past intersects an open neighbourhood in $L$ of $L \cap \mathcal{J}_{+}$. This is not true for $S$ unless the system had been radiating all the way down to the infinite past $\mathrm{I}_{-}$. This is to show that for systems capable of radiating off energy and momentum it is generally inconsistent to require $\operatorname{supp}(\mathbf{T}) \cap \Sigma$ to be compact for spacelike $\Sigma$, unless $\Sigma$ is a Cauchy surface. 
recent [28], replace our hypothesis of stationarity, i.e., $\partial T^{\alpha \beta} / \partial x^{0}=0$, by the stronger hypothesis of staticity, which in addition to stationarity also contains the three conditions $\mathrm{T}^{0 \mathrm{~m}}=0$. Systems satisfying this condition of staticity, as well as local energy-momentum conservation (3), are called complete static systems by Laue. For them Laue derives the result that the integrals (4) form the components of a "four-vector". We shall clarify the mathematical content of this statement below.

Many text-book derivations suffer from inaccuracies, taking sufficient conditions also as necessary, or unduly neglecting the role of the boundary integrals. Quite recently, a pedagogically guided attempt has been made to bring some logical order into the various classic textbook statements of what Laue's theorem actually says, as well as the corresponding derivations [28]. Unfortnunately the derivation given in [28] is itself logically incomplete ${ }^{5}$ and entirely phrased in a non-geometric component language, so that it remains entirely unclear what becomes of Laue's theorem in curved spacetimes.

Finally we stress once more that the requirement (22) should not be confused with that in which $\Sigma$ is also acted on by $\Lambda$ :

$$
\mathrm{P}^{\alpha}[\Lambda \cdot \Sigma, \Lambda \cdot \mathbf{T}]=\Lambda_{\beta}^{\alpha} \mathrm{P}^{\beta}[\Sigma, \mathbf{T}],
$$

where $\Lambda \cdot \Sigma$ denotes the image of the hyperplane $\Sigma$ under the (active!) Lorentz transformation $\Lambda$. This equation is trivially satisfied for all $\mathbf{T}$, as one easily sees by a simple change-or-variable transformation of the integral and the fact that now not only the components $T^{\alpha \beta}$ but also the $n_{\beta}$ transforms so that $T^{\alpha \beta} n_{\beta}$ already transforms as a four vector. Hence no further conditions on $\mathbf{T}$ result. Equation (23) is a trivial requirement that merely states that $\mathrm{P}^{\alpha}$ has been constructed from geometric objects. But this is not the point! Rather, the point is whether the quantities $\mathrm{P}^{\alpha}$ behave like a four vector under boosts which exclusively act on the matter fields so as to change their state relative to a fixed background structure. The difference between (22) and (23) is easily overlooked if one adopts a passive interpretation of Lorentz transformations, in which case one is tempted to let $\Lambda$ act on any geometric structure that appears in one's formulae ("anything that has indices on it is transformed"). This confusion has led to several fake-resolutions of the transformation problem in the classic literature, like e.g. in [21] and [22].

\subsection{The many uses of Laue's theorem}

Before we outline the geometric theory, let us say a few words on the many uses of Laue's theorem. Laue himself applied it to many of the at first sight paradoxical results in special-relativistic kinematics, most importantly to the null results of the classic 1903 experiment by Trouton and Noble [18]. All these apparent paradoxa result from applications of (15) to energy-momentum tensors violating the hypotheses of Laue's theorem, so that the space integrals in these equations do not vanish. As an easy first example consider the case when the space integrals in (15c) (i.e.

\footnotetext{
5 The incompleteness occurs in the third line of the string of equations (6) on p. 1471 of [28], where the integration domain is changed from a hyperplane $t^{\prime}=$ const to a tilted hyperplane $t=$ const. This step in [28] does not follow from the initial hypothesis of $t$-independence, unless some form of local energy-momentum conservation is imposed in addition, which at this point would amount to a petitio principii.
} 
over the longitudinal-transversal spatial components of $\mathbf{T}$ ) do not vanish. This implies that as a result of a pure boost the systems picks up momentum perpendicular to the boost direction. Momentum and velocity of such a boosted system will no longer be aligned. This also means that putting such a system into the boosted state will necessitate the transmission of angular momentum, i.e. the action of torque. Another, historically earlier apparent paradox concerns the Coulomb field of charge distributions. Assuming spatial isotropy, the tracelessness of the electromagnetic energy-momentum tensor $\left(T^{00}=T^{11}+T^{22}+T^{33}\right)$ gives $\int_{\Sigma} T^{11}=\frac{1}{3} P^{0}$. Hence, even if $\mathrm{P}^{1}=0$, we have $\overline{\mathrm{P}}^{0}=\frac{4}{3} \gamma \mathrm{P}^{0}$ and $\overline{\mathrm{P}}^{1}=\frac{4}{3} \beta \gamma \mathrm{P}^{0}$, which looks all right except for the factor 4/3. Systems to which this applies are, e.g., the Coulomb field outside a sphericallysymmetric surface charge distribution (like in "classical models of the electron"; see, e.g., [22]), or the black-body radiation inside a container. The reason why all these examples violate the hypotheses of Laue's theorem is, in Laue's terminology, that they are not "complete" in the following sense: They either do not satisfy (3) or, if the points of $\Sigma$ where (3) is violated are removed from $\Sigma,\left.\mathbf{T}\right|_{\partial \Sigma} \neq 0$, i.e. the boundary conditions are not met. For example, including the stresses that are needed to prevent the charge distribution of the "classical electron" (so-called Poincaré stresses, because in [20] Poincaré already pointed out the necessity of such - possibly non-electromagnetic - stresses) from exploding, or the black-body radiation from escaping the cavity, removes all these paradoxa in a general and model-independent fashion. Therein, as well as in its universal applicability, lies the strength of Laue's theorem.

We end this section by a more modern application of Laue's theorem. Let us consider the question of whether an electromagnetically bound system, like a large molecule, obeys a simple version of Einstein's equivalence principle, according to which the system's (centre of mass) acceleration in a given static and weak gravitational field is independent of internal energies. As we restrict to weak and static fields, we write for the components of the space-time metric

$$
g_{\alpha \beta}=\eta_{\alpha \beta}+h_{\alpha \beta} \text {, }
$$

where $\left\{\eta_{\alpha \beta}\right\}=\operatorname{diag}(1,-1,-1,-1)$ represents the flat Minkowski background and $h_{\alpha \beta}$ deviations from it, which are considered small, which means higher powers than the first of $h_{\alpha \beta}$, as well as of its first two derivatives, are neglected. The linearized Einstein equations for static Newtonian sources (where only the 00 component of the energy-momentum tensor is assumed non-zero) then give, as is well known,

$$
h_{\alpha \beta}(\vec{x})=\delta_{\alpha \beta} \frac{2 \Phi(\vec{x})}{c^{2}} .
$$

Here $\Phi$ is the standard Newtonian potential and $\delta_{\alpha \beta}$ the Kronecker delta (not the Minkowski metric!). The coupling of a the weak gravitational field $h_{\alpha \beta}$ to the energy momentum tensor of our system (molecule) is given by the interaction Lagrangian

$$
\mathrm{L}_{\text {int }}=\frac{1}{2} \int_{\Sigma} d^{3} x \mathrm{~T}^{\alpha \beta} \mathrm{h}_{\alpha \beta}=\frac{1}{\mathrm{c}^{2}} \int_{\Sigma} d^{3} \chi \Phi\left(\mathrm{T}^{00}+\mathrm{T}^{11}+\mathrm{T}^{22}+\mathrm{T}^{33}\right) .
$$

If, in addition, we assume that the external gravitational potential $\Phi$ is approximately constant over the spatial support of $T^{\alpha \beta}$, and that the spatial support at time $t=0$ is centred about the 
spatial position $\vec{z}$ (e.g. some Newtonian centre of mass), we get

$$
\mathrm{L}_{\text {int }}(\vec{z})=\Phi(\vec{z}) \int_{\Sigma} \mathrm{d}^{3} x\left(\mathrm{~T}^{00}+\mathrm{T}^{11}+\mathrm{T}^{22}+\mathrm{T}^{33}\right) / \mathrm{c}^{2}
$$

It is clear now that if $\mathbf{T}$ satisfies the hypotheses of Laues theorem only the first integral survives and $\mathrm{L}_{\mathrm{int}}(\vec{z})$ equals the inertial mass of the system times the Newtonian potential. This implies the weak equivalence principle since it explicitly demonstrates the equality of the inertial and passive gravitational mass (i.e. that mass to which the interaction with an external gravitational field is proportional to). Here we note in passing that the combination $T^{00}+T^{11}+T^{22}+T^{33}$ equals $2\left(T^{\alpha \beta}-\frac{1}{2} \eta^{\alpha \beta} \eta_{\mu \nu} T^{\mu \nu}\right) n_{\alpha} n_{\beta}$ and corresponds to the integrand of the so-called Tolman mass [26]. We refer to section 9 of the arXiv version of [11] for a discussion of how the Tolman mass relates to other mass concepts, like the Komar mass.

Let us for the moment forget about Laue's theorem and proceed further in analyzing (27). Our material system that is subject to the weak gravitational field consists of charged particles interacting via their Coulomb interactions (we shall neglect radiation). Hence $\mathbf{T}=\mathbf{T}_{1}+\mathbf{T}_{2}$, with subscript 1 referring to the particles and subscript 2 to the electromagnetic field. For a particle at rest the space integral of $T_{1}^{00}$ equals $m_{0} c^{2}$ and all other components of vanish. From (12) we then infer that a single slowly moving particle (in $x^{1}$ direction) has to leading order in $v / \mathrm{c}$ a $T_{1}^{00}$ space integral of $m_{0} c^{2}+E_{k i n}$, a $T_{1}^{11}$ space integral of $2 E_{k i n}$, and all other diagonal components vanishing (to leading order). The sum over all particles then gives, to leading order in $v / c$,

$$
\int_{\Sigma} d^{3} x T_{1}^{00}=M_{0} c^{2}+E_{k i n}, \quad \int_{\Sigma} d^{3} x \delta_{a b} T_{1}^{a b}=2 E_{k i n},
$$

where $M_{0}$ is the sum of all rest-masses over all particles and $E_{\text {kin }}$ is the sum of all kinetic energies over all particles.

On the other hand, the electromagnetic field has a trace-free energy momentum tensor and hence

$$
\int_{\Sigma} d^{3} x T_{2}^{00}=U, \quad \int_{\Sigma} d^{3} x \delta_{a b} T_{2}^{a b}=U
$$

where $\mathrm{U}$ is the total energy stored in the electromagnetic field, which we here identify with the total Coulombian binding energy, after having subtracted the diverging Coulombian self-energy of each particle (compare footnote 6 below).

Hence we get as passive gravitational mass for our molecular matter $M_{0}+\left(3 E_{k i n}+2 U\right) / c^{2}$. But we would have expected $M_{0}+\left(E_{\text {kin }}+U\right) / c^{2}$ and now wonder what the excess of $\left(2 E_{\text {kin }}+\right.$ $\mathrm{U}) / \mathrm{c}^{2}$ might mean. Well, from our derivation we know that this combination corresponds to the space integral of $\delta_{a b}\left(T_{1}^{a b}+T_{2}^{a b}\right)$, which has to vanish if $\mathbf{T}=\mathbf{T}_{1}+\mathbf{T}_{2}$ satisfies the hypotheses of Laue's theorem (as we assume). But suppose we had never heard of Laue's theorem, what could have rescued us from concluding that there is something seriously wrong with the equivalence principle? Precisely this situation came up in an investigation of Carlip's [4], who concluded that it is the virial theorem that rescues us. Recall that in the low-velocity ("non-relativistic") approximation with homogeneous potentials of degree -1 , that is, any combination of attractive 
and repulsive $1 / r$ potentials, the virial theorem just implies that $2 E_{\text {kin }}+U=0$ for the timeaverages of kinetic and potential energies. ${ }^{6}$ Indeed, that is how we could have concluded without invoking Laue's theorem. But I think the foregoing discussion has made it clear how elegant and forceful Laue's theorem really is. In fact, there is a relation between the derivation of Laue's theorem given above and the relativistic virial theorem given in [16], in the sense that the virial theorem is a proper implication of Laue's theorem, in that it only states the vanishing of the spatial integral of the spatial trace of $\mathbf{T}$, not the vanishing of the spatial integrals of each component of $\mathbf{T}$ separately (except $\mathrm{T}^{00}$ ).

\section{Geometric theory}

With this second section, which also contains six subsections, we turn to the second half of our programme as outlined in the introduction. Having discussed Laue's theorem and its physical relevance in the first section, we now wish to know how to formulate it in a modern differentialgeometric language. Such a formulation should in particular make clear the geometric hypotheses underlying it. These hypotheses are not at all obvious from the component-based "derivation" given above, which uses integrals over tensor components and identities like (18), none of which make any proper geometric sense a priori, and only receive their restricted meaning in the context of SR through the affine and metric structure of Minkowski space. In this section we shall give a proper geometric statement and proof of a generalisation of Laue's theorem that is valid outside SR and reduced to the classical statement given above if suitably specialised. Nothing of what we are now going to say depends crucially on the number $n$ of dimensions of $M$, so that we shall leave it open. $g$ is then a Lorentzian metric on $M$ of signature $(1, n-1)$, i.e. "mostly minus" (compare Appendix B.4). Indices are now written in the latin alphabet ranging from 0 to $n-1$.

6 It is important here to note the following point: In electrostatics, where (in SI-units) $\varepsilon_{0} \Delta \phi=-\rho, \phi$ being the scalar potential satisfying $\vec{E}=-\vec{\nabla} \phi$ and $\rho$ being the charge distribution, the electric interaction energy for any charge distribution $\rho$ of compact support can be written in the following equivalent forms:

$$
E_{\text {int }}=\frac{\varepsilon_{0}}{2} \int d^{3} x\|\vec{E}(\vec{x})\|^{2}=\frac{1}{8 \pi \varepsilon_{0}} \iint d^{3} x d^{3} x^{\prime} \frac{\rho(\vec{x}) \rho\left(\vec{x}^{\prime}\right)}{\left\|\vec{x}-\vec{x}^{\prime}\right\|} .
$$

The first expression shows that $\mathrm{E}_{\text {int }}$ is always positive and cannot possibly be identified with $\mathrm{U}$ in the virial theorem, where due to $2 \mathrm{E}_{\text {kin }}+\mathrm{U}=0$ and $2 \mathrm{E}_{\text {kin }} \geq 0$ we clearly must have $\mathrm{U} \leq 0$. On the other hand, if we tried to apply (30) to a number $n$ of point-particles, where $\rho(\vec{x})=\sum_{a=1}^{n} q_{a} \delta^{(3)}\left(\vec{x}-\vec{x}_{a}\right)$, E $E_{\text {int }}$ would clearly diverge due to the self-interaction of each individual particle. The second expression in (30) then shows that after subtraction of each diverging part the remaining finite part is

$$
E_{\text {int }}^{(\text {finite })}=\frac{1}{4 \pi \varepsilon_{0}} \sum_{a<b} \frac{q_{a} q_{b}}{\left\|\vec{x}_{a}-\vec{x}_{b}\right\|}
$$

where the sum runs over all $\frac{1}{2} n(n-1)$ possible combinations for $a, b \in\{1, \cdots, n\}$ for which $a<b$. That regularised expression $E_{\text {int }}^{(\text {finite })}$ may well assume negative values for a mixture of positively and negatively charged particles and it is that expression that we may identify with $U$ in the virial theorem for such mixed collections of charged particles; compare $\S 34$ of [16]. 


\subsection{Alternative representation of energy-momentum distributions}

Instead of $\mathbf{T} \in \Gamma(T M \vee T M)$ we define a new object by pulling down the first index on $\mathbf{T}$ and Hodge-dualising on the second:

$$
\mathcal{T}:=\mathrm{T}_{\mathrm{ab}} \theta^{\mathrm{a}} \otimes \star \theta^{\mathrm{b}} \in \Gamma\left(\mathrm{T}^{*} \mathrm{M} \otimes \bigwedge^{\mathrm{n}-1} \mathrm{~T}^{*} M\right) .
$$

$\mathcal{T}$ can be considered as a $T^{*} M$-valued $(n-1)$-form, or equivalently, as an $(n-1)$-form-valued linear map on TM.

We assume $\mathbf{T}$ to be "complete" in the sense of Laue's, by which we mean (2), i.e. the vanishing of the vector field $\nabla \cdot \mathbf{T}:=\nabla_{\mathrm{b}} \mathrm{T}^{\mathrm{ab}} \partial / \partial x^{\mathrm{a}}$ (in $\mathrm{n}$ dimensions latin indices range from 0 to $\mathrm{n}-1$ ). Using the exterior covariant derivative $\mathrm{D}$, the following identity holds:

$$
\mathrm{D} \mathcal{T}=(\nabla \cdot \mathbf{T})^{b} \otimes \varepsilon .
$$

Here $\varepsilon$ is the volume $n$-form associated to $g$ and $b$ denotes again the "index-lowering isomorphism" defined by g (compare (90)).

Let us give a proof of (33) in component language. For that we recall that the exterior covariant derivative $D$ of an $r$-form $F$ with values in a vector bundle $V$ over $M$, i.e. of $F \in \Gamma\left(V \otimes \Lambda^{r} T^{*} M\right)$, is obtained from an ordinary torsion-free covariant derivative $\nabla: \Gamma\left(\mathrm{V} \otimes \Lambda^{\mathrm{r}} \mathrm{T}^{*} \mathrm{M}\right) \rightarrow \Gamma(\mathrm{V} \otimes$ $\mathrm{T}^{*} \mathrm{M} \otimes \bigwedge^{\mathrm{r}} \mathrm{T}^{*} \mathrm{M}$ ) (which in our case is the Levi-Civita connection) by total antisymmetrisation in $\mathrm{T}^{*} \mathrm{M} \otimes \bigwedge^{\mathrm{r}} \mathrm{T}^{*} \mathrm{M}$, so as to obtain a map $\mathrm{D}: \Gamma\left(\mathrm{V} \otimes \Lambda^{\mathrm{r}} \mathrm{T}^{*} \mathrm{M}\right) \rightarrow \Gamma\left(\mathrm{V} \otimes \Lambda^{\mathrm{r}+1} \mathrm{~T}^{*} \mathrm{M}\right)$. More precisely, just writing the form-components (and keeping the $\mathrm{V}$-valuedness implicit), we have $(D F)_{a_{0} \cdots a_{r}}=(r+1) \nabla_{\left[a_{0}\right.} F_{\left.a_{1} \cdots a_{r}\right]}$. Here the antisymmetrisation bracket $[\cdots]$ is as in (89). The reason for the factor $(r+1)$ is the very same as for the corresponding formula for the ordinary exterior derivative $d$, which in turn is just (88) applied to $p=1$ and $q=r$. Hence, using (99) and (107) with $n_{-}=(n-1)$ (recall our "mostly-minus" convention for the signature in $n$ dimensions), we indeed derive (33) in component form through the following lines:

$$
\begin{aligned}
& (\mathrm{DT})_{\mathrm{ab}_{1} \cdots \mathrm{b}_{\mathfrak{n}}}=\mathrm{ng} \mathrm{g}_{\mathrm{ac}} \nabla_{\left[\mathrm{b}_{1}\right.} \mathrm{T}_{\left.\mathrm{b}_{2} \cdots \mathrm{b}_{\mathfrak{n}}\right]}^{\mathrm{c}} \\
& =n g_{a c} \nabla_{\left[b_{1}\right.} T^{c b} \varepsilon_{\left.|b| b_{2} \cdots b_{n}\right]} \\
& =-(n / n !) g_{a c} \varepsilon_{b_{1} \cdots b_{n}} \varepsilon^{c_{1} c_{2} \cdots c_{n}} \nabla_{c_{1}} T^{c b} \varepsilon_{b_{2} \cdots c_{n}} \\
& =g_{\mathrm{ac}} \varepsilon_{\mathrm{b}_{1} \ldots \mathrm{b}_{\mathrm{n}}} \nabla_{\mathrm{b}} \mathrm{T}^{\mathrm{cb}} \text {. }
\end{aligned}
$$

We conclude from (33) that (2) is equivalent to $\mathcal{T}$ having vanishing exterior covariant derivative: $\mathrm{D} \mathcal{T}=0$. However, this does not yet define a conservation law. For that we need an ordinary d-closed $(n-1)$-form. The recipe to get this from a D-closed $T^{*} M$-valued $(n-1)$ form is to contract the value of the latter with a vector field $K \in \Gamma(T M)$; for that contraction we write

$$
\mathcal{T}_{\mathrm{K}}:=i_{\mathrm{K}} \mathcal{T}
$$

Using that $\mathrm{D}$ and $\mathrm{d}$ coincide on ordinary forms, a straightforward calculation, very similar indeed to that in (34), then shows

$$
\mathrm{d} \mathcal{T}_{\mathrm{K}}=\mathrm{D}\left(\mathfrak{i}_{\mathrm{K}} \mathcal{T}\right)=\mathfrak{i}_{\mathrm{K}}(\mathrm{D} \mathcal{T})+\mathbf{T}\left(\nabla \mathrm{K}^{b}\right) \varepsilon
$$


Here the function $\mathbf{T}\left(\nabla K^{b}\right)$ is the contraction of $\mathbf{T} \in \Gamma(T M \vee T M)$ with $\nabla K^{b} \in \Gamma\left(T^{*} M \otimes T^{*} M\right)$, i.e. in components $T^{a b} \nabla_{a} K_{b}$. Hence, given symmetry (1) and $D \mathcal{T}=0$ (i.e. (3)), $\mathcal{T}_{K}$ is closed for general $\mathbf{T}$, if and only if the symmetric part of $\nabla \mathrm{K}^{b}$ vanishes. Now, as $\nabla$ is the Levi-Civita covariant derivative with respect to $\mathrm{g}$, we have the well known formula that twice the symmetric part of $\nabla K^{b}$ equals $L_{K} g$, the Lie derivative of $g$ with respect to $K$; in components ${ }^{7}$

$$
\nabla_{\mathrm{a}} \mathrm{K}_{\mathrm{b}}+\nabla_{\mathrm{b}} \mathrm{K}_{\mathrm{a}}=\left(\mathrm{L}_{\mathrm{k}} \mathrm{g}\right)_{\mathrm{ab}} .
$$

In other words, given (1) and (3), the $(n-1)$-form $\mathcal{T}_{K}$ is closed if and only if $K$ is a Killing vector field, i.e. a vector field whose flow is by isometries of $(M, g)$. It is at this point that both conditions (1) and (3) enter our discussion and further consequences in an essential fashion.

\subsection{On the notions of "charges" and "conservation"}

In physics the closedness of the $(n-1)$-form $\mathcal{T}_{K}$ is often expressed synonymously by "conservation" (i.e. divergencelessness) of the "current" (i.e. vector field)

$$
\mathrm{J}_{\mathrm{K}}:=\left(\star \mathcal{T}_{\mathrm{K}}\right)^{\sharp}=\mathrm{K}_{\mathrm{a}} \mathrm{T}^{\mathrm{ab}} \partial / \partial x^{\mathrm{b}} .
$$

Then

$$
\mathrm{d} \mathcal{T}_{\mathrm{K}}=0 \Leftrightarrow \nabla \cdot \mathrm{J}_{\mathrm{K}}=0 .
$$

Note that $\sharp$ is the inverse of $b$ (compare (90)) and also that $\star 0 \star$ is the identity on one- and $(n-1)$-forms according to (102) and (107) for mostly-minus Lorentz signatures.

The physical concepts of charges and their conservation directly derive from formulae like (39) as follows: Integrate $0=d \mathcal{T}_{K}$ over a $n$-dimensional submanifold $\Omega \subset M$ with piecewise smooth boundary $\partial \Omega=\Sigma_{1} \cup \Sigma_{2} \cup B$, where $B$ is such that $\mathcal{T}_{K}$ restricted to $B$ vanishes; then, by Stokes' theorem,

$$
\int_{\Sigma_{1}} \mathcal{T}_{\mathrm{K}}=-\int_{\Sigma_{2}} \mathcal{T}_{\mathrm{K}}=\int_{-\Sigma_{2}} \mathcal{T}_{\mathrm{K}}
$$

Here $\left(-\Sigma_{2}\right)$ denotes $\Sigma_{2}$ endowed with opposite orientation. If, as in the usual argument, $\Sigma_{1}$ and $\Sigma_{2}$ are two spacelike submanifolds whose intersection with $\operatorname{supp}(\mathbf{T})$ is compact and B is a timelike cylinder connecting the boundaries of $\Sigma_{1}$ and $\Sigma_{2}$ such that $B \cap \operatorname{supp}(\mathbf{T})=\emptyset$, then in physics we say that the "charge" associated with the conserved current $\mathrm{J}_{\mathrm{K}}$, which is defined to be the flux of $J_{K}$ through the hypersurface, is conserved in the sense of being independent of the spacelike hypersurface it is integrated over. Note that the flux of $J_{K}$ is obtained by integrating $\star\left(J_{K}^{b}\right)$.

We summarise all this in the following definition, in which we drop the subscript $\mathrm{K}$ on $\mathrm{J}$ since it applies to all $J \in \Gamma(T M)$, independently of whether or not they derive from $\mathbf{T}$ by the construction above.

\footnotetext{
7 This formula has additional terms involving the non-metricity tensor $\mathrm{Q}:=\nabla \mathrm{g}$ and the torsion for general connections.
} 
Definition 1. Let $\Omega \subset M$ be a n-dimensional submanifold with piecewise smooth boundary $\partial \Omega=\Sigma_{1} \cup \Sigma_{2} \cup \mathrm{B}$ such that $\mathrm{B} \cap \operatorname{supp}(\mathrm{J})=\emptyset$, then we call $\Sigma_{1}$ and $\Sigma_{2}$ homologous modulo $\operatorname{supp}(J)$.

Definition 2. Let $\mathrm{J} \in \Gamma(\mathrm{TM})$ be any vector field and $\Sigma \subset M$ an $(\mathrm{n}-1)$-dimensional oriented submanifold. Then we call

$$
\mathrm{Q}(\Sigma, \mathrm{J}):=\int_{\Sigma} \star \mathrm{J}^{\mathrm{b}}
$$

the flux of J through $\Sigma$ or, equivalently, the charge of J at $\Sigma$.

Corollary 1. If $\mathrm{J} \in \Gamma(\mathrm{TM})$ is divergenceless (is a "conserved current") and $\Sigma_{1}$ and $\Sigma_{2}$ are homologous modulo $\operatorname{supp}(\mathrm{J})$, that is, if there is an $\mathrm{n}$-dimensional submanifold $\Omega \subset \mathrm{M}$ with piecewise smooth boundary $\partial \Omega=\Sigma_{1} \cup \Sigma_{2} \cup \mathrm{B}$ where $\operatorname{supp}(\mathrm{J}) \cap \mathrm{B}=\emptyset$, then

$$
\mathrm{Q}\left(\Sigma_{1}, \mathrm{~J}\right)=-\mathrm{Q}\left(\Sigma_{2}, \mathrm{~J}\right)=\mathrm{Q}\left(-\Sigma_{2}, \mathrm{~J}\right)
$$

where $-\Sigma_{2}$ stands for $\Sigma_{2}$ endowed with opposite orientation.

Proof. This is an immediate consequence of Stokes' theorem.

The charges $\mathrm{Q}(\Sigma, J)$ are just numbers associated to vector fields $J$ and hypersurfaces $\Sigma$. In physics, these numbers receive their interpretation through the interpretation of $J$ and their significance through additional special properties of J, most importantly its vanishing divergence and certain constraints on its support. Corollary 1 states to what extent these numbers are independent of $\Sigma$. This leads to the concept of "conservation" in the sense that the charges measured at different boundaries are the same. If one of these boundaries is regarded as time evolution of the other, then we obtain the ordinary notion of charge conservation "in time".

\subsection{Group actions and the habitat of charges}

To a large degree independent of the issue of conservation is the issue of interpretation. As already stated, the interpretation of the charges must follow from the interpretation of J. In our case, the vector fields $J$ we consider derive from energy-momentum tensors $\mathbf{T}$ and some preferred vector field $\mathrm{V}$. Hence the interpretation of charge is essentially connected with $\mathrm{V}$ : Which vector field $V$ are we using in order to turn $\mathbf{T}$ into a "current" $J=J_{V}:=i_{V} \mathbf{T}$, the charge of which we consider? That is the question we address in this subsection. We know that if $\mathrm{V}=\mathrm{K}$ is Killing then $\mathrm{J}_{\mathrm{K}}$ is covariant divergenceless and the charges are conserved. But for the time being we wish to be independent of that assumption and hence shall not assume $V$ to be Killing.

Vector fields $V \in \Gamma(T M)$ generate diffeomorphisms of $M$, which we may physically interpret as "motions" if acting on geometric objects on M representing physical systems. Of "motions" we usually think as being composable and forming a group. It is the group structure of a set of motions that we usually employ in order to interpret it elements. For example, in Newtonian mechanics we characterise an overall motion of a system of point particles as being either a 
"translation" or "rotation", depending on how this particular motion sits inside the group $E_{3}$ of euclidean motions that we think of as having been implemented as acting on space (in this case by isometries). In $E_{3}$ translations are invariantly characterised as elements of the maximal abelian normal subgroup. That is, translations form a unique 3-dimensional subgroup in the 6-dimensional group $E_{3}$. In contrast, there is no unique rotation subgroup in $E_{3}$. Rather, $E_{3}$ contains a 3-parameter family of different copies of rotation subgroups corresponding to the different choices of origin in space about which one rotates (that is kept fixed unter all rotations). These different copies are all related by conjugation with translations (shifting the origin). Hence we may not speak of "the" but only of "a" rotation subgroup in $E_{3}$, each one corresponding to its own origin in space.

Similar remarks apply to the Poincaré group in special-relativitsic physics. (Compare Appendix A.5 and [12] for a more detailed discussion of the semi-direct product structure of the Poincaré group in terms of a unique subgroup of translations and a non-unique complementary subgroup of Lorentz transformations.) Here again "translations" in space and time are invariantly characterised as forming the maximal abelian normal subgroup of the Poincaré group. If the translation is timelike we call the corresponding charge "energy" and "linear momentum" if it is spacelike. Charges corresponding to any of the Lorentz subgroups, of which there is an n-parameter family, are then associated to, e.g., angular momentum or centre-of-mass motion. Again picking one of the Lorentz subgroups is equivalent to picking a point in spacetime, the "origin" left fixed by all Lorentz transformations.

Let us now turn to the general case and capture the situation by supposing that a finitedimensional Lie group $G$ acts on the left on the manifold $M$. The words "left action" mean that we have a homomorphism of groups ( $e \in G$ is the group identity):

$$
\Phi: \mathrm{G} \rightarrow \operatorname{Diff}(M), \quad \mathrm{g} \mapsto \Phi_{\mathrm{g}}, \quad \Phi_{e}=\mathrm{id}_{M}, \quad \Phi_{\mathrm{g}} \circ \Phi_{\mathrm{h}}=\Phi_{\mathrm{gh}} .
$$

For a "right action" the last equation would be replaced by $\Phi_{g} \circ \Phi_{h}=\Phi_{h g}$, but we shall stick to the "left" convention.

Later we will consider special actions $\Phi$ of $G$ on $M$ which act by isometries with respect to a given metric $g$ on $M$. This means that

$$
\Phi_{h}^{*} g=g
$$

for each $h \in G$. But for the time being we shall keep the discussion independent of that assumptions as long as possible. Hence we stress that all statements below do not assume the action to be isometric unless explicitly stated so.

Any group-homomorphism between Lie-groups induces a homomorphism between the corresponding Lie-algebas, which is just given by the differential of the former at the group identity. The Lie-algebra of $\operatorname{Diff}(M)$ is given by the infinite-dimensional vector space $\Gamma(T M)$ of vector fields on $M$ whose Lie bracket is minus the vector-field commutator. Hence we have

$$
\mathrm{V}: \operatorname{Lie}(\mathrm{G}) \rightarrow \Gamma(\mathrm{TM}), \quad \mathrm{V}_{\xi}(\mathrm{p}):=\left.\frac{\mathrm{d}}{\mathrm{ds}}\right|_{s=0} \Phi_{\exp (\mathrm{s} \xi)}(\mathrm{p})
$$


which satisfies

$$
\begin{aligned}
-\left[\mathrm{V}_{\xi}, \mathrm{V}_{\zeta}\right] & =\mathrm{V}_{[\xi, \zeta]}, \\
\left(\Phi_{g}\right)_{*} \mathrm{~V}_{\xi} & =\mathrm{V}_{\mathrm{Ad}_{\mathrm{g}}(\xi)} .
\end{aligned}
$$

We refer to the Appendix of [12] or the appendix of the arXiv version of [11] for a detailed proof. Before we continue, we give $V_{\xi}$ a proper name:

Definition 3. Given an action $\Phi$ of $\mathrm{G}$ on $\mathrm{M}$, then $\mathrm{V}_{\xi} \in \Gamma(\mathrm{TM})$ is called the fundamental vector field for that action on $\mathrm{M}$ corresponding to $\xi \in \mathrm{Lie}(\mathrm{G})$.

Note that the bracket $[\cdot, \cdot]$ on the left-hand side of $(44 \mathrm{~b})$ is the vector-field commutator, which is minus the Lie-bracket in the Lie-algebra of $\operatorname{Diff}(M)$, whereas the $[\cdot, \cdot]$ on the right-hand side of (44b) is the Lie-bracket in $\operatorname{Lie}(G)$. This is the reason why there is an additional minus sign on the left-hand side of (44b) that makes this relation a Lie homomorphism. ${ }^{8}$

Given $\mathbf{T}$ we can for each fundamental vector field $V_{\xi} \in \Gamma(T M)$ and each oriented ( $n-$ 1)-dimensional submanifold $\Sigma$ calculate the charge $\mathrm{Q}(\Sigma, J)$ according to (41). The result is a number that depends linearly on $\xi$ since it is obtained by composing three linear maps:

$$
\operatorname{Lie}(\mathrm{G}) \ni \xi \mapsto \mathrm{V}_{\xi} \mapsto \mathcal{T}_{V_{\xi}} \mapsto \int_{\Sigma} \mathcal{T}_{V_{\xi}} \in \mathbb{R} .
$$

In other words, it defines an element in $\operatorname{Lie}^{*}(G)$, the vector space dual to $\operatorname{Lie}(G)$. This assignment of an element in $\operatorname{Lie}^{*}(G)$ obviously depends on $\Sigma$ and $\mathbf{T}$, but as $\mathbf{T}$ depends on the underlying physical fields, we shall regard the dependence to be on $\Sigma$ and some physical fields. Which physical fields? Here we shall make the same distinction as in our previous discussion in Section 1.3. In the more general geometric setting that we consider now, we assume $\mathbf{T}$ and hence $\mathcal{T}$ to be build locally ${ }^{9}$ from a set of fields $\mathrm{F} \in \Gamma(\mathrm{B})$ whose energy-momentum distribution is represented by $\mathbf{T}$ (or $\mathcal{T}$ ), and possibly a set of complementary background fields $\mathrm{F}^{\prime} \in \Gamma\left(\mathrm{B}^{\prime}\right)$. Here, $B$ and $B^{\prime}$ denote some natural bundles over $M$. Following standard terminology (compare Appendix B.3) the word "natural" is added here in order to indicate our requirement that any diffeomorphism $\Phi$ of $M$ shall have a natural action on sections in $B$ and $B^{\prime}$, which we denote by $\mathrm{D}$ and $\mathrm{D}^{\prime}$ respectively. We then assume the generalisation of (10), which now reads

$$
\mathcal{T}\left(\mathrm{D}_{\Phi} \mathrm{F}, \mathrm{D}_{\Phi}^{\prime} \mathrm{F}^{\prime}\right)=\left(\Phi^{-1}\right)^{*} \mathcal{T}\left(\mathrm{F}, \mathrm{F}^{\prime}\right) .
$$

It says that $\mathcal{T}$ evaluated on the $\Phi$ transformed fields $\mathrm{D}_{\Phi} \mathrm{F}$ and $\mathrm{D}_{\Phi}^{\prime} \mathrm{F}^{\prime}$ is the $\Phi$-transform of $\mathcal{T}\left(F, F^{\prime}\right)$. Here we already took into account that the $\Phi$-transform of a section in $T^{*} M \otimes$ $\bigwedge^{(n-1)} T^{*} M$ is the pull-back with the inverse $\Phi^{-1}$. Condition (46) may be seen as a completeness condition, in the sense that we did in fact consider all fields $\left(F, F^{\prime}\right)$ on which $\mathcal{T}$ truly depends. This is the generalisation of (10) referred to at the end of Section 1.3. As already stressed

8 Sometimes this minus sign is said to express a Lie-anti-homomorphism, namely whenever Lie $(\operatorname{Diff}(M))$ is endowed with the opposite Lie structure, in which the Lie-bracket is the commutator of vector fields. The minus sign in (44b) would also disappear if we considered a right rather than left action of $G$ on $M$, though then other minus signs would pop up elsewhere. See the appendix in [12] for a detailed discussion and more information.

9 Meaning that the value of $\mathcal{T}$ at a point $p \in M$ depends on the values of $F$ and $F^{\prime}$ and at most finitely many derivatives at $p$. 
there, in the context of general groups $G$ acting on $M$ it becomes particularly important to distinguish between the systems whose states are represented by $F$ and whose energy-momentum distribution is fully captured by $\mathcal{T}$, and the wider system whose states are represented by $\left(F, F^{\prime}\right)$ on which $\mathcal{T}$ may actually also depend.

Definition 4. The map, that according to (45) assigns an element in $\mathrm{Lie}^{*}(\mathrm{G})$ to each triple $\left(\Sigma, F, F^{\prime}\right)$ is called momentum map ${ }^{10}$ and denoted by $\mathfrak{M}$. We shall put the arguments in square brackets and write $\mathfrak{M}\left[\Sigma, \mathrm{F}^{\prime} \mathrm{F}^{\prime}\right] \in \mathrm{Lie}^{*}(\mathrm{G})$ since it may take another argument from $\mathrm{Lie}(\mathrm{G})$ to turn that element into a real number. For $\xi \in \operatorname{Lie}(G)$ we then write $\mathfrak{M}\left[\Sigma, F, F^{\prime}\right](\xi) \in \mathbb{R}$ and note that this number is just the flux of $\mathrm{J}_{V_{\xi}}=i_{V_{\xi}} \mathbf{T}$ through $\Sigma$ :

$$
\mathfrak{M}\left[\Sigma, F, F^{\prime}\right](\xi):=Q\left(\Sigma, i_{V_{\xi}} \mathbf{T}\left(F, F^{\prime}\right)\right)=\int_{\Sigma} \mathcal{T}_{\mathcal{V}_{\xi}}\left(F, F^{\prime}\right) \text {. }
$$

We wish to know how the value in $\operatorname{Lie}^{*}(G)$ of the momentum map $\mathfrak{M}$ behaves under the action of the group $\mathrm{G}$. That is answered by

Theorem 2. Let $(G, \Phi, M)$ be a left action of the finite dimensional Lie group $G$ on $M$ and $\mathcal{T} \in \Gamma\left(\mathrm{T}^{*} \mathrm{M} \otimes \bigwedge^{(\mathrm{n}-1)} \mathrm{T}^{*} \mathrm{M}\right)$ a section depending locally on $\left(\mathrm{F}, \mathrm{F}^{\prime}\right) \in \Gamma(\mathrm{B}) \times \Gamma\left(\mathrm{B}^{\prime}\right)$ so that (46) is valid for all $\Phi=\Phi_{g}, g \in \mathrm{G}$; then

$$
\mathfrak{M}\left[\Phi_{g} \Sigma, D_{\Phi_{g}} F, D_{\Phi_{g}}^{\prime} F^{\prime}\right]=\operatorname{Ad}_{g}^{*}\left(\mathfrak{M}\left[\Sigma, F, F^{\prime}\right]\right),
$$

where $\Phi_{g} \Sigma$ is the Image of $\Sigma$ under $\Phi_{g}$ and $\mathrm{Ad}^{*}$ denotes the co-adjoint representation of $\mathrm{G}$ on $\mathrm{Lie}^{*}(\mathrm{G})$, i.e. the inverse-transposed of the adjoint representation.

Proof. The proof follows from a string of elementary steps:

$$
\begin{aligned}
\mathfrak{M}\left[\Phi_{g} \Sigma, D_{\Phi_{g}} F, D_{\Phi_{g}}^{\prime} F^{\prime}\right](\xi) & \stackrel{1}{=} \int_{\Phi_{g} \Sigma} \mathcal{T}_{V_{\xi}}\left(D_{\Phi_{g}} F, D_{\Phi_{g}}^{\prime} F^{\prime}\right) \\
& \stackrel{2}{=} \int_{\Phi_{g} \Sigma} i_{V_{\xi}} \mathcal{T}\left(D_{\Phi_{g}} F, D_{\Phi_{g}}^{\prime} F^{\prime}\right) \\
& \stackrel{3}{=} \int_{\Phi_{g} \Sigma} i_{V_{\xi}}\left(\Phi_{g^{-1}}^{*} \mathcal{T}\left(F, F^{\prime}\right)\right) \\
& \stackrel{4}{=} \int_{\Phi_{g} \Sigma} \Phi_{g^{-1}}^{*}\left(\mathcal{T}_{\Phi_{g^{-1}} V_{\xi}}\left(F, F^{\prime}\right)\right) \\
& \stackrel{5}{=} \int_{\Sigma} \mathcal{T}_{V_{A_{g^{-1}}}(\xi)}\left(F, F^{\prime}\right) \\
& \stackrel{6}{=} \mathfrak{M}\left[\Sigma, F, F^{\prime}\right]\left(\operatorname{Ad}_{g^{-1}}(\xi)\right) \\
& \stackrel{7}{=} \operatorname{Ad}_{g}^{*}\left(\mathfrak{M}\left[\Sigma, F, F^{\prime}\right]\right)(\xi) .
\end{aligned}
$$

\footnotetext{
${ }^{10}$ The momentum map is a standard tool in symplectic geometry and Hamiltonian Mechanics; see, e.g., Chapter 4.2 of [1] and references given therein. The difference between our and the standard definition is that we start from the energy-momentum tensor, which we assume to be given, without explicitly using the symplectic geometry of phase space.
} 
Steps 1 and 2 just follow the definitions, step 3 uses (46), step 4 uses the general relation $i_{X} \circ$ $\Phi^{*}=\Phi^{*} \circ i_{\Phi_{*} X}$ for the pull-back and the insertion map applied to forms, which is obvious from the fact that the pull-back and push-forward are relatively transposed maps, step 5 uses (44c) and the general relation ("change-of-variable formula") $\int_{\Sigma} \phi^{*} \alpha=\int_{\phi(\Sigma)} \alpha$ for the integration of a $k$ form over a k-dimensional submanifold, step 6 (like step 1) invokes the definition of $\mathfrak{M}$, and, finally, step 7 just uses the definition of the co-adjoint representation.

We stress the generality of (48), which in particular does not put any further constraint on the action of $G$ on $M$ other than the validity of (46), in particular we did so far not require the action to be isometric. Such further conditions now come into play if we want to strengthen (48) similarly to the way (9) strengthens (10) and (22) strengthens (23). That is, we wish to state (48) with $G$ just acting on the dynamical fields $F$ and not on $\Sigma$ and the background fields $F^{\prime}$. Hence we wish to have

$$
\mathfrak{M}\left[\Sigma, D_{\Phi_{g}} F, F^{\prime}\right]=\operatorname{Ad}_{g}^{*}\left(\mathfrak{M}\left[\Sigma, F, F^{\prime}\right]\right) .
$$

This is the generalisation and, in fact, proper phrasing of the requirement that that global "momenta" calculated from a local distribution of dynamical fields $F$ transform as "vectors" under the action of a group $G$ on $M$ (a statement to be qualified below).

So we ask: under what conditions is it true that (48) implies (50)? We start with the dependence on $F^{\prime}$ and observe that $D_{\Phi_{g}}^{\prime} F^{\prime}$ may be replaced by $F^{\prime}$ if $D_{\Phi_{g}}^{\prime}$ acts trivially on the background fields $F^{\prime}$, i.e., if $G$ is a symmetry group for all configurations $F^{\prime}$. This is trivially always the case if the set of background fields $F^{\prime}$ is empty, i.e. if the energy-momentum tensor exclusively only depends on the fields the energy momentum distribution of which it represents. This will be hard to achieve in general. More common is the case in which it depends at least on the background metric $\mathrm{g}$, like, e.g., in the traditional (metric) formulation of Maxwell's electrodynamics. Without external sources no other $F^{\prime}$ exist, so $F^{\prime}=g$ and $D_{\Phi_{h}}^{\prime} F^{\prime}=\left(\Phi_{h}^{-1}\right)^{*} g$, which equals $g$ for all $h \in G$ if and only if $G$ acts on $M$ isometrically with respect to $g$. With external sources the background fields $\mathrm{F}^{\prime}$ also include charge densities and currents, which then, too, must remain invariant under the action of $G$.

Next we turn to the dependence on $\Sigma$. We note that the integral (47) would be the same for two hypersurfaces $\Sigma_{1}$ and $\Sigma_{2}$ if they were homologous modulo $\operatorname{supp}(\mathbf{T})$ and if $\mathcal{T}_{V_{\xi}}$ were closed for all $\xi \in \operatorname{Lie}(G)$. In view of (36) a necessary and sufficient condition for the latter to hold is that $V_{\xi}$ is Killing, i.e. that $G$ acts as isometries on $(M, g)$. From that we infer

Corollary 2. The momentum map $\mathfrak{M}$ satisfies the restricted condition of equivariance in the form (50) if $\mathrm{G}$ acts on $(\mathrm{M}, \mathrm{g})$ by isometries, if $\mathrm{G}$ is a symmetry of all other background fields $\mathrm{F}^{\prime}$ (other than the metric), and if $\Phi_{\mathrm{h}} \Sigma$ is homologous to $\Sigma$ modulo $\operatorname{supp}(\mathbf{T})$ for all $\mathrm{h} \in \mathrm{G}$.

Note that the hypotheses of this corollary turn the values of the momentum map and hence the charges (contractions of $\mathfrak{M}$ with elements of $\operatorname{Lie}(G)$ ) into "conserved" charges, in the sense that the charges at all $\Phi_{h} \Sigma$ are the same. If the group $G$ of motions contain time translations, the general notion of "conserved" used here reduces to the usual "constant in time".

At the end of this section we wish to stress that the hypotheses on mentioned in corollary 2 are sufficient conditions, which need not be necessary in special situations. We made some effort to 
first derive the general condition of equivariance (48) under comparatively weak hypotheses, and then showed how the standard conditions (like that $\mathrm{G}$ acts isometrically) lead to the restricted condition of equivariance (50), known as $\mathrm{Ad}^{*}$-equivariance.

\subsection{Poincaré covariant momenta}

The equivariance condition (50) gives the precise expression to the statement usually employed in physics, that conserved momenta, i.e. the values of $\mathfrak{M}$, behave "covariantly" under a certain group of motions. At the same time we learn how to characterise the vector space of which the conserved momenta are members of. They are elements in $\operatorname{Lie}^{*}(G)$ and they transform under the co-adjoint representation of $\mathrm{G}$.

The physical interpretation of these momenta must be obtained from G. If, e.g., G is the group of space-time translations, which is invariantly contained in the Poincare group as the maximal abelian normal subgroup, then the corresponding "momenta" correspond to energy and linear momentum. Together they transform like "four vectors" under Poincaré transformations, that is, trivially under translations and by the defining representation of the Lorentz group. But that last statement is deceptive, because our momenta are really elements of $\operatorname{Lie}^{*}(G)$, not the fourdimensional real vector space $V$ underlying affine Minkowski space. In this subsection we show how this is properly phrased for the Poincaré group and how it relates to our previous discussion in Sections 1.4 and 1.5.

To start the discussion, let $\mathrm{G}=$ Poin be the Poincaré group of spacetime, which is isomorphic to $V \rtimes$ Lor, where Lor is the Lorentz group, so that for $(a, A)$ and $(b, B) \in V \rtimes$ Lor, we have

$$
(a, A)(b, B)=(a+A b, A B)
$$

and

$$
(a, A)^{-1}=\left(-A^{-1} a, A^{-1}\right),
$$

where juxtapositions like $A b$ denote the action of $A \in$ Lor on $b \in V$ under the defining representation. We refer to Appendix A for a discussion of the (non natural!) isomorphism Poin $\cong \mathrm{V} \rtimes$ Lor. From (51) we can easily calculate the adjoint and co-adjoint representation on $\mathrm{Lie}($ Poin) and Lie*(Poin) respectively. These take a convenient form if we identify both of these linear spaces with $\mathrm{V} \oplus(\mathrm{V} \wedge \mathrm{V})$, which also allows for an easy comparison between the adjoint and co-adjoint representations (which are now realised on the same vector space). Recall that usually one identifies Poin $\cong V \rtimes$ Lor and Lor with isometries of $(V, \eta)$. This induces the usual identification $\operatorname{Lie}($ Poin $) \cong V \rtimes \operatorname{Lie}(\operatorname{Lor})$ where $\operatorname{Lie}(\operatorname{Lor}) \cong\{X \in \operatorname{End}(V)$ : $\eta(X v, w)=-\eta(v, X w) \forall v, w \in V\}$. To that we add the identification of the latter (the $\eta$ antisymmetric endomorphisms) with $\mathrm{V} \wedge \mathrm{V}$, in that we agree that $x \wedge y \in \mathrm{V} \wedge \mathrm{V}$ corresponds to the following element of $\operatorname{End}(V): x \wedge y(v):=x \eta(y, v)-y \eta(x, v)$, which one easily checks is $\eta$-antisymmetric. The extension of that correspondence to all elements in $V \wedge V$ (not just pure exterior products) then follows from linearity. Hence the Lie brackets between to elements $(x, X)$ and $(y, Y)$ in $V \oplus(V \wedge V) \cong \operatorname{Lie}($ Poin $)$ is

$$
[(x, X),(y, Y)]=(X \cdot y-Y \cdot x,[X, Y]),
$$


where the action of $X, Y \in V \wedge V$ on $V$ (denoted by a dot in (52)) is as explained above, and $[X, Y]:=X \circ Y-Y \circ X$ is just the commutator of these maps. Finally, we identify Lie* $(G)$ with $\mathrm{V} \oplus(\mathrm{V} \wedge \mathrm{V})$ by identifying the dual space of $\mathrm{V} \oplus(\mathrm{V} \wedge \mathrm{V})$ with itself through the following inner product (non-degenerate symmetric bilinear form) on $V \oplus(\mathrm{V} \wedge \mathrm{V})$ :

$$
\langle(x, X),(y, Y)\rangle=\eta(x, y)+\frac{1}{2} \eta \otimes \eta(X, Y) .
$$

Here the second term just corresponds to what we called the renormalised inner product on forms in equation (95) of the Appendix. Hence if $X=u \wedge v=u \otimes v-v \otimes u$ and $Y=w \wedge z=$ $w \otimes z-z \otimes w$ we have $\frac{1}{2} \eta \otimes \eta(X, Y)=\eta(u, v) \eta(v, z)-\eta(u, z) \eta(v, w)$.

The adjoint and co-adjoint representation on $\mathrm{V} \oplus(\mathrm{V} \wedge \mathrm{V})$ are now easily obtained as follows. Let $s \mapsto(b(s), B(a))$ be a curve in Poin through the identity at $s=0$. We set with $\mathrm{d} /\left.\mathrm{d} s\right|_{s=0}(\mathrm{~b}(\mathrm{~s}), \mathrm{B}(\mathrm{s}))=(\mathrm{P}, \mathrm{M}) \in \mathrm{V} \oplus(\mathrm{V} \wedge \mathrm{V})$ and have

$$
\begin{aligned}
\operatorname{Ad}_{(a, A)}(P, M) & :=\left.\frac{d}{d s}\right|_{s=0}(a, A)(b(s), B(s))(a, A)^{-1} \\
& =(A P-[(A \otimes A) M] a,(A \otimes A) M) .
\end{aligned}
$$

The co-adjoint representation is the inverse-transposed of that, transposition now being understood in terms of the inner product (53):

$$
\left\langle\left(P^{\prime}, M^{\prime}\right), \operatorname{Ad}_{(a, A)}(P, M)\right\rangle=\left\langle\operatorname{Ad}_{(a, A)}^{\top}\left(P^{\prime}, M^{\prime}\right),(P, M)\right\rangle .
$$

Writing this out using (53) we read off (dropping the primes)

$$
\operatorname{Ad}_{(a, A)}^{\top}(P, M)=\left(A^{-1} P,\left(A^{-1} \otimes A^{-1}\right) M+\left(A^{-1} a\right) \wedge\left(A^{-1} P\right)\right),
$$

and therefore

$$
\begin{aligned}
\operatorname{Ad}_{(a, A)}^{*}(P, M) & :=\left(\operatorname{Ad}_{\left(-A^{-1} a, A^{-1}\right)}\right)^{\top}(P, M) \\
& =(A P,(A \otimes A) M-a \wedge A P) .
\end{aligned}
$$

The first entry on the right-hand side of (57) shows what previously was meant when we said that $\mathrm{P}=\mathrm{P}^{\alpha} e_{\alpha}$ transformed as a "four vector". But note from the second slot that $M$ does not just transform as an "antisymmetric 2nd-rank tensor", there is an additional piece a $\wedge A P$ expressing the base-point dependence of angular momentum and centre-of-mass motion, which group-theoretically exists as a result of the fact that Lor $\subset$ Poin is not a normal subgroup and hence $\operatorname{Lie}($ Lor $) \subset \operatorname{Lie}($ Poin) not an ideal. 


\subsection{Laue's theorem: geometric formulation}

We are now in a position to phrase Laue's theorem in geometric language and show what its underlying assumptions are. We start with the remark that this theorem is, in fact, a statement about conserved currents which is then specialised to the case that the current is constructed from an energy-momentum tensor through contraction with a Killing field. So let us start by assuming that we have a conserved current $J \in \Gamma(T M)$, i.e. in components $J=J^{\alpha} \partial / \partial x^{\alpha}$ with $\nabla_{\alpha} J^{\alpha}=0$. Its corresponding $(n-1)$-form, $\star J^{b} \in \Gamma\left(\bigwedge^{(n-1)} T^{*} M\right)$ will be called $\mathcal{J}$. Hence $\mathcal{J}$ is closed (compare Appendix B.2),

$$
\mathrm{d} \mathcal{J}=0 .
$$

We assume $\mathcal{J}$ to admit a symmetry, that is, we assume there exists a vector field $U \in \Gamma($ TM) such that the Lie derivative of $\mathcal{J}$ with respect to $U$ vanishes:

$$
\mathrm{Lu}_{\mathrm{u}}=0 \text {. }
$$

This is equivalent to saying that the motions generated by $\mathrm{U}$ (its flow) leave $\mathcal{J}$ invariant.

Next we recall Cartan's formula, according to which the Lie derivative of forms is given by the symmetrised combination of the exterior derivative $d$ and the map $i_{u}$ which inserts $U$ in the first tensor slot:

$$
\mathrm{L}_{\mathrm{u}}=\mathrm{d} \circ i_{\mathrm{u}}+i_{\mathrm{u}} \circ \mathrm{d} .
$$

Hence (58) and (59) imply that $\mathcal{J}_{\mathrm{u}}:=i_{\mathrm{u}} \mathcal{J} \in \Gamma\left(\bigwedge^{(\mathrm{n}-2)} \mathrm{T}^{*} M\right)$ is closed

$$
\mathrm{d} \mathcal{J} \mathrm{u}=0 \text {. }
$$

Clearly that implies by Stokes' theorem that if $\sigma_{1}, \sigma_{2} \subset M$ are $(n-2)$-dimensional submanifolds which are homologous modulo $\operatorname{supp}(\mathrm{J})$ then the integrals of $\mathcal{J}_{\mathrm{u}}$ over $\sigma_{1}$ equals that of $-\sigma_{2}$. But is not quite yet the result we are after. Suppose $\varphi \in C^{\infty}(M)$ is a smooth function, then $\mathrm{d} \varphi \wedge \mathcal{J}_{\mathrm{u}} \in \Gamma\left(\bigwedge^{(\mathrm{n}-1)} \mathrm{T}^{*} \mathrm{M}\right)$ is not only closed but exact (compare Appendix B.2):

$$
\mathcal{J}_{(\varphi, \mathrm{U})}:=\mathrm{d} \varphi \wedge \mathcal{J}_{\mathrm{u}}=\mathrm{d}\left(\varphi \mathcal{J}_{\mathrm{u}}\right) .
$$

Now, this leads us to the sought-for generalised Laue-type theorem for conserved currents:

Theorem 3 (Laue's theorem, geometric version for currents). Let $(\mathrm{M}, \mathrm{g})$ be a $\mathrm{n}$-dimensional Semi-Riemannian manifold, $\mathrm{J} \in \Gamma(\mathrm{TM})$ a divergenceless (with respect to the Levi-Civity connection) vector field, and $\mathcal{J} \in \Gamma\left(\bigwedge^{(n-1)} \mathrm{T}^{*} \mathrm{M}\right)$ its corresponding closed $(\mathrm{n}-1)$-form. Let Further $\mathrm{U} \in \Gamma(\mathrm{TM})$ be a vector field generating symmetries of $\mathcal{J}$, i.e. $\mathrm{Lu} \mathcal{J}=0$. Then, for any $\varphi \in \mathrm{C}^{\infty}(\mathrm{M})$ and any $(\mathrm{n}-1)$-dimensional submanifold $\Sigma \subset M$ such that $\partial \Sigma \cap \operatorname{supp}\left(\mathcal{J}_{(\varphi, \mathrm{U})}\right)=\emptyset$ or sufficiently rapid fall-off of $\varphi \mathcal{J}$ at each end of $\Sigma$, we have

$$
\begin{aligned}
0 & =\int_{\Sigma} \mathcal{J}_{(\varphi, \mathrm{U})} \\
& =\int_{\Sigma}\left(\mathrm{U}(\varphi) \star \mathrm{J}^{\mathrm{b}}-\mathrm{J}(\varphi) \star \mathrm{U}^{\mathrm{b}}\right) \\
& =\int_{\Sigma}(\mathrm{U}(\varphi) \mathrm{g}(\mathrm{J}, \mathrm{n})-\mathrm{J}(\varphi) \mathrm{g}(\mathrm{U}, \mathrm{n})) \mathrm{d} \mu_{\Sigma} \quad(\Sigma \text { non lightlike })
\end{aligned}
$$


Here $\mathcal{J}_{\mathrm{u}}:=\mathfrak{i}_{\mathrm{u}} \mathcal{J}$ and $\mathcal{J}_{(\varphi, \mathrm{u})}$ as in (62). Moreover, $\mathrm{n}$ denotes the non-null normal to $\Sigma$ and the volume form $\mathrm{d} \mu_{\Sigma} \in \Gamma\left(\bigwedge^{(\mathrm{n}-1)} \mathrm{T}^{*} \Sigma\right)$ on $\Sigma$ is defined by $\mathrm{d} \mu_{\Sigma}= \pm i_{n} \varepsilon$ with positive/negative sign for timelike/spacelike $\mathrm{n}$.

Proof. The first equality (63a) follows immediately from the exactness of $\mathcal{J}_{(\varphi, \mathrm{U})}$, Stokes' theorem and the hypothesis that $\mathcal{J}_{(\varphi, \mathrm{U})}$ vanishes on the boundary of $\Sigma$ or falls-off sufficiently fast at each end. The second expression (63b) follows from $i_{\mathrm{u}}(\mathrm{d} \varphi \wedge \mathcal{J})=\mathrm{U}(\varphi) \mathcal{J}-\mathcal{J}_{(\varphi, \mathrm{U})}$ and the definition of $\star$, given in (98b) of the Appendix, applied to the left-hand side: $i_{u}(d \varphi \wedge \mathcal{J})=$ $\mathfrak{i}_{\mathrm{u}}\left(\mathrm{d} \varphi \wedge \star \mathrm{J}^{\mathrm{b}}\right)=\left(\mathfrak{i}_{\mathrm{u}} \varepsilon\right)\left\langle\mathrm{d} \varphi, J^{b}\right\rangle_{\text {norm }}=J(\varphi) \star \mathrm{U}^{b}$. Finally, the third expression (63c) is valid only in the case the normal $n$ to $\Sigma$ is nowhere lightlike, for then (109) gives $\star J^{b}=i_{\mathcal{J}} \varepsilon$ and hence $\left.\star J^{b}\right|_{T \Sigma}=\left.(g(J, n) / g(n, n)) i_{n} \varepsilon\right|_{T \Sigma}=g(J, n) d \mu_{\Sigma}$ with $d \mu_{\Sigma}:=\left.g(n, n) i_{n} \varepsilon\right|_{T \Sigma}$. Note that $g(n, n)= \pm 1$, depending on whether $n$ is timelike/spacelike ( $\Sigma$ spacelike/timelike).

\subsection{Recovery of the classical statement of Laue's theorem}

The theorem above is very general, in that for given $J$ and $\mathrm{U}$ it holds for all $\Sigma$ and $\varphi$. In order to see that the classical version of Laue's theorem emerging as a special case, we now explicitly list all the particular choices leading to it:

1. $(M, g)$ is 4-dimensional Minkowski space, i.e. there exist global affine coordinates $\left\{x^{0}, x^{1}, x^{2}, x^{3}\right\}$ so that

$$
g=\eta=\eta_{\alpha \beta} d x^{\alpha} \otimes d x^{\beta} \quad \text { with } \quad \eta_{\alpha \beta}=\operatorname{diag}(1,-1,-1,-1) \text {. }
$$

2. The symmetry-generating vector field $U$ for $J$ is that generating time translations, so that

$$
\mathrm{Lu}_{\mathcal{U}}=0 \quad \text { where } \quad \mathrm{U}=\frac{\partial}{\partial x^{0}} .
$$

Note that since $\frac{\partial}{\partial x^{0}}$ is also a symmetry of $g=\eta$ the condition $L_{u} \mathcal{J}=0$ is equivalent to $\mathrm{LuJ}_{\mathrm{J}}=0$ (because $\mathrm{L}_{\mathrm{u}}$ commutes with index lowering/raising and also with the Hodge duality map).

3. We take $\Sigma:=\left\{p \in M: x^{0}(p)=0\right\}$ so that

$$
\mathrm{n}=\frac{\partial}{\partial x^{0}} \quad \text { and } \quad \mathrm{d} \mu_{\Sigma}=\mathrm{d} x^{1} \wedge \mathrm{d} x^{2} \wedge \mathrm{d} x^{3}=: \mathrm{d}^{3} x
$$

Given (1-3) the integrand in (63c) becomes (we write $\partial \varphi / \partial x^{\alpha}=: \varphi_{, \alpha}$; latin indices range from 1 to 3 ; note that $\mathrm{J}_{0}=\mathrm{J}^{0}$ since $\left.\eta_{00}=1\right)$ :

$$
\mathrm{U}(\varphi) \mathrm{g}(J, \mathrm{n})-\mathrm{J}(\varphi) \mathrm{g}(\mathrm{U}, \mathrm{n})=\varphi_{, 0} \mathrm{~J}_{0}-\mathrm{J}^{\alpha} \varphi_{, \alpha}=-\mathrm{J}^{\mathrm{a}} \varphi_{, \mathrm{a}} .
$$

Hence, in this special case, Theorem 3 just reduces to a more or less obvious result. Indeed, if $J_{, 0}^{\alpha}=0$ then $J_{, \alpha}^{\alpha}=J_{, a}^{a}$ so that the spatial components define a 3-dimensional divergenceless vector field in space which vanishes if integrated against any gradient vector field, provided that the surface terms one encounters in partial integration vanish. 
So far we have not restricted to any special choice of $\varphi$, except that the product $\varphi J$ must vanish on $\partial \Sigma$ or fall-off sufficiently rapidly at $\Sigma$ 's ends. These conditions can of course always be met by appropriate choices of $\varphi$, given any J. But if J already has support bounded away from $\partial \Sigma$ and/or falls off sufficiently fast at each end of $\Sigma$, then we may even multiply it with unbounded functions $\varphi$, like any of the spatial coordinate functions $x^{\mathrm{m}}$, and obtain

$$
\int_{\Sigma} d^{3} x J^{a}\left(x^{m}\right)_{, a}=\int_{\Sigma} d^{3} x J^{m}=0
$$

for all $m \in\{1,2,3\}$. Not that in this formula the components of $J$ must refer to affine (inertial) coordinates.

Now, the last step in order to arrive at our previous classic formulation of Laue's theorem in Section 1.5 is to apply this to the conserved current

$$
\mathrm{J}=\mathrm{JK}_{\mathrm{K}}:=\mathfrak{i}_{\mathrm{K}^{b}} \mathbf{T}=\mathrm{K}_{\alpha} \mathbf{T}^{\alpha \beta} \frac{\partial}{\partial x^{\beta}},
$$

where K is Killing. Since we are in Minkowski space we have 10 Killing fields at our disposal, but in order to reproduce equation (17) we just need to consider the space-time translations

$$
K=\frac{\partial}{\partial x^{\mu}} .
$$

Indeed, inserting (67a) and (67b) into (66) immediately leads to (17).

This derivation of the original result (17) might look like cracking a walnut with a sledgehammer. However, looking at the $3+2$ equations (64) and (67) make it clear that this is a very special case indeed and that the general result it derived from, i.e. (63), is far more general. Also, the derivation of (17) from (63) makes it clear what geometric structures are necessary in order to make sense of the original form. The physical interpretation and significance of other consequences of (63) remain to be developed. One could, e.g., think of stationary axisymmetric spacetimes in which $\mathrm{U}$ is taken to be the timelike and $\mathrm{K}$ the rotational Killing field (which commute, $[\mathrm{U}, \mathrm{K}]=0)$. A stationary $\mathbf{T}$, i.e. $\mathrm{L}_{\mathrm{U}} \mathbf{T}=0$, then implies $\mathrm{L}_{\mathrm{U}} \mathcal{J}=0$ with $\mathcal{J}=\star\left(i_{\mathrm{K}^{b}} \mathbf{T}\right)^{b}$, as required. I do not know of attempts to evaluate the consequences of (63) in such situations. At the same time is is likely that such consequences are implicit in some of the works on the dynamics of matter in stationary axisymmetric spacetimes, like Kerr. 


\section{Conclusions and Outlook}

The underlying theme of this investigation consists in the problem of how local distributions of energy and momentum meaningfully combine ("add") to global quantities. In Section 2.3 we gave an answer that required a global group action on the state space of the physical system under consideration. The group and its action links the local geometric objects with the global quantities whose habitat and interpretation are closely related to group-theoretic concepts. If the action of the group on state space derives from an action on space-time, the global quantities receive their interpretation from the type of motions generated by the group. This is encoded in the momentum map of Definition 4, together with its property (50) of restricted Ad*equivariance under motions of the dynamical fields alone. Only in combination with restricted $\mathrm{Ad}^{*}$-equivariance can we properly associate the local dynamical fields $\mathrm{F}$ with globally conserved quantities and identify their mathematical habitat. For that it is essential that the representatives of physical states, i.e. the local fields F, are geometric objects in the sense that they are naturally acted upon by diffeomorphisms. ${ }^{11}$ Without such a group-theoretic link no obvious meaning can generally be given to integrals over local energy-momentum distributions.

Even though we only considered matter fields in fixed background space-times (which may or may not satisfy Einstein's equations), our considerations can be extended to GR where the metric field itself is dynamical and contributes to the global quantities that are usually identified with energy and momentum. In that context presumably the first person to seriously wonder about the habitat and transformation properties of formally constructed global quantities was Felix Klein in [14], who coined the term "free affine vector" to characterise the four numbers that resulted from integrations of components of local geometric objects (involving the metric and the connection). The word "free" and "affine" were meant to say that these four numbers are the component of a vector, but that vector is not attached to any point in spacetime. The word "vector" was meant to indicate that these four numbers transform properly under a restricted class of diffeomorphisms. The latter were defined as those preserving the metric structure at large spatial distances, which was assumed to be Minkowskian. Hence, modulo those diffeomorphisms that tend to the identity at spacelike infinity and which are considered mere gauge transformations (redundancies in the description), the Poincare group should result with all its associated conserved charges. Hence the idea is to construct a momentum map for the Poincaré group in GR as well, given that the field configurations satisfy suitable asymptotic conditions. This idea is to a certain extend realised by the so-called ADM constructions, as explained in more detail in [7] and very lucidly in [2] (see also [11] for a general review of the ADM construction, its relation to momentum maps, and all the relevant references). Again, and now for the last time, we stress that such expressions loose their physical interpretation if formally transcribed to situations without a proper group-theoretic context. This, it seems to me, is often not sufficiently appreciated.

\footnotetext{
${ }^{11}$ A pragmatic definition of a local geometric object on a manifold is that for any chart it may be fully represented in the chart domain by arrays of numbers, with well defined transformation rules under changes of charts; see, e.g., $\S 4.13$ of Trautmann's lecture [27]. This comprises all sections in bundles associated to the bundle of linear frames, including tensors and their densities, which all transform linearly, and also connections, which change by affine transformations. For a more systematic approach to define geometric structures, see, e.g., [24].
} 


\section{Appendices}

The following three appendices combine general information on our notation and conventions in differential geometry, as well as some basic but conceptually important structural properties of Minkowski space and its automorphism group, the Poincaré group.

\section{A. Minkowski space and Poincaré group}

Within the realm of Special Relativity, spacetime is modelled by Minkowski space. In this appendix we wish to briefly recall some of the mathematical structures underlying Minkowski space and its automorphism group, called the Poincare group. A proper awareness of these structures is important for the discussion in the main text.

For the sake of generality we shall give our discussion for spacetimes of general dimension $n$. This will not induce any additional complications. Now, $n$-dimensional Minkowski space is defined to be a real affine space of dimension $n \geq 2$ whose associated vector space is endowed with a non-degenerate symmetric bilinear form of signature $(1, n-1)$ [i.e. one positive, $n-1$ negative dimensions], also called a Lorentzian inner product. Minkowski space is also a real ndimensional Manifold homeomorphic to $\mathbb{R}^{n}$ with a Lorentzian metric and associated Levi-Civita connection which is flat.

\section{A.1. Affine spaces}

Here we wish to concentrate on the first definition, which immediately connects to the basic physical idea of "inertial motion", i.e. the motion of force-free extensionless test particles, which is represented by the straight-lines in affine space. We recall that an $n$-dimensional affine space is a triple $(M, V,+)$, where $M$ is a set, $V$ is an $n$-dimensional real vector space and the familiar symbol + denotes an action of $V$ - considered as abelian group - on the set $M$ which is simply transitive. The word "action" means that there is a map $\mathrm{M} \times \mathrm{V} \rightarrow \mathrm{M}$ denoted by $(\mathrm{m}, v) \mapsto \mathrm{m}+v$ such that $\mathrm{m}+0=\mathrm{m}$ for all $\mathrm{m} \in M$ and $\mathrm{m}+\left(v+v^{\prime}\right)=(\mathrm{m}+v)+v^{\prime}$. Note that in this last equation the first $(+)$-sign on the left-hand side denotes $V$ 's action on $M$ whereas the second denotes vector addition. On the right-hand side both $(+)$-signs denote V's action on $M$. The word "transitive" means that for each pair $\left(m, m^{\prime}\right) \in M \times M$ there exists a $v \in \mathrm{V}$ such that $\mathrm{m}^{\prime}=\mathrm{m}+v$. The word "simply" then further requires that this $v \in \mathrm{V}$ be unique. Given that, we may extend our suggestive notation and write $v=m^{\prime}-m$ for that unique element $v \in V$ which, when acting on $m$, gives $m^{\prime}$. Hence we may write equations like $\left(m^{\prime}-p\right)+(p-m)=m^{\prime}-m$ or $m+\left(m^{\prime}-p\right)=m^{\prime}+(m-p)$, valid for all $m^{\prime}, m, p$ in $M$.

To sum up, we may "add" vectors to points in affine space to get new point in affine space, and also "subtract" two points in affine space to get a vector. These two operations behave in a natural "associative" way as exemplified by the equations above. But note that points in affine 
space cannot be added. It is true that every point $o \in M$ defines a bijection

$$
\phi_{\mathrm{o}}: M \rightarrow \mathrm{V}, \quad \mathrm{m} \mapsto \phi_{\mathrm{o}}(\mathrm{m}):=\mathrm{m}-\mathrm{o} .
$$

One might use $\phi_{\mathrm{o}}$ to pull back the linear structure from $V$ to $M$, so that "addition" in $M$ could be defined by $m+m^{\prime}:=\phi_{o}^{-1}\left(\phi_{o}(m)+\phi_{o}\left(m^{\prime}\right)\right)=0+(m-o)+\left(m^{\prime}-o\right)$. However, the result of "adding" $m$ to $m^{\prime}$ in this fashionthen then depends on the choice of o. In fact, any $p \in M$ could be made the result of adding $m$ to $m^{\prime}$ : Just choose $o=m+\left(m^{\prime}-p\right)=m^{\prime}+(m-p)$.

\section{A.2. Affine automorphisms and affine groups}

An affine automorphisms of an affine space is a bijection $F: M \rightarrow M$ of the following form: There exists a point $m \in M$ and a map $f \in G L(V)$ (here and below GL(V) denotes the general linear group of $V$, i.e. the group of all linear invertible self-maps of $V)$ ), such that $F$ has the form

$$
F(m+v)=F(m)+f(v)
$$

It immediately follows that once (69) is true for some $m$ and some $f$ then it also holds for all $m \in M$ with the same $f$. Hence $f$ is uniquely determined by $F$. Therefore, if $m^{\prime}+v^{\prime}=m+v$, we may either write $F\left(m^{\prime}\right)+f\left(v^{\prime}\right)$ or $F(m)+f(v)$ for the image point.

Affine maps form a group under composition which we denote by $\operatorname{Aff}(M, V,+)$. Given two affine maps $F$ and $F^{\prime}$ with associated linear maps $f$ and $f^{\prime}$, the composition $F^{\prime} \circ F$ has associated linear map $f^{\prime} \circ f$ as is easily checked. Moreover, if $F=i d_{M}$ then $f=i d_{V}$. Hence the map

$$
\begin{aligned}
\pi: \operatorname{Aff}(M, V,+) & \rightarrow \mathrm{GL}(\mathrm{V}) \\
\mathrm{F} & \mapsto \pi(\mathrm{F})=\mathrm{f},
\end{aligned}
$$

which assigns to any affine map $F$ its associated linear map $f$, is a surjective homomorphism of groups. The kernel of $\pi$ is given by an abelian normal subgroup $\operatorname{Trans}(M, V,+) \subset A f f(M, V,+)$ which one calls the translations of $M$ and which is naturally isomorphic with $V$. The quotient $\operatorname{Aff}(M, V,+) / \operatorname{Trans}(M, V,+)$ is isomorphic to $\operatorname{GL}(V)$. In fact, $\operatorname{Aff}(M, V,+)$ is isomorphic to the semi-direct product $V \rtimes G L(V)$ in which GL(V) acts as automorphism of the group $V$ by its defining representation. This means that for $(a, A)$ and $(b, B)$ in the set $V \times G L(V)$, their multiplication and inversion in $\mathrm{V} \rtimes \mathrm{GL}(\mathrm{V})$ is

$$
\begin{aligned}
(a, A)(b, B) & =(a+A b, A \circ B), \\
(a, A)^{-1} & =\left(-A^{-1} a, A^{-1}\right) .
\end{aligned}
$$

It is important to understand how this isomorphism between $\operatorname{Aff}(M, V,+)$ and $V \rtimes \operatorname{GL}(V)$ comes about, because it is not natural due to the fact that it depends on the choice of an auxiliary point $\mathrm{o} \in M$. Given such a point $\mathrm{o}$, a bijection $\Phi_{\mathrm{o}}$ between the sets $\operatorname{Aff}(M, V,+)$ and $\mathrm{V} \times \mathrm{GL}(\mathrm{V})$ can be obtained by a combination of the maps (68) and (70) as follows:

$$
\begin{aligned}
\Phi_{\mathrm{o}}: \operatorname{Aff}(\mathrm{M}, \mathrm{V},+) & \rightarrow \mathrm{V} \times \mathrm{GL}(\mathrm{V}) \\
\mathrm{F} & \mapsto \Phi_{\mathrm{o}}(\mathrm{F}):=\left(\phi_{\mathrm{o}}(\mathrm{F}(\mathrm{o})), \pi(\mathrm{F})\right) .
\end{aligned}
$$


Given two elements $F, F^{\prime}$ in $\operatorname{Aff}(M, V,+)$, we write $\Phi_{o}(F)=(v, f)$ and $\Phi_{o}\left(F^{\prime}\right)=\left(v^{\prime}, f^{\prime}\right)$ and have $\Phi_{o}\left(F^{\prime} \circ F\right)=\left(v^{\prime}+f^{\prime}(v), f^{\prime} \circ f\right)$, as a short computation shows. This means that the bijection (72) becomes an isomorphism of groups if $V \times G L(V)$ is endowed with the semi-direct product structure (71). Note that both, $\mathrm{V}$ as well as GL(V), are subgroups of $\mathrm{V} \times \mathrm{GL}(\mathrm{V})$. $\mathrm{V}$ is the invariant subgroup $\operatorname{kernel}(\pi)$ and $\mathrm{GL}(\mathrm{V})$ is the (non-invariant) subgroup of those elements fixing the point $o \in M$. Hence, in contrast to the translations, the linear part $G L(V)$ has no natural place in $\operatorname{Aff}(M, V,+)$.

\section{A.3. Affine bases and affine charts}

An affine basis $\mathrm{B}$ of an affine space consists of a tuple $\mathrm{B}=(\mathrm{o}, \mathrm{b})$, where $\mathrm{o} \in \mathrm{M}$ and $\mathrm{b}=$ $\left\{e_{1}, \cdots e_{n}\right\}$ is a basis of $V$. It defines a bijection of sets $\phi_{B}: M \rightarrow \mathbb{R}^{n}$ through $\phi_{B}(m):=$ $\left(x^{1}(m), \cdots, x^{\mathfrak{n}}(m)\right)$, where $x^{a}(m):=\theta^{a}(m-o)$. The inverse map $\phi_{B}^{-1}: \mathbb{R}^{n} \rightarrow M$ is given by $\phi_{B}^{-1}\left(x^{1}, \cdots, x^{\mathfrak{n}}\right)=0+x^{a} e_{a}$. The bijection $\phi_{B}$ becomes a topological homeomorphism if the topology given to $M$ is the initial one, i.e. the weakest (coarsest) to make $\phi_{B}$ continuous (the continuity of $\phi_{\mathrm{B}}^{-1}$ follows from the invariance-of-domain theorem). Moreover, $\phi_{\mathrm{B}}$ defines a global chart (i.e. an atlas with a single chart) which can be used to endow $M$ with the structure of a differentiable manifold. We call such a chart an affine chart. Given two different affine bases, $\mathrm{B}=(\mathrm{o}, \mathrm{b})$ and $\mathrm{B}^{\prime}=\left(\mathrm{o}^{\prime}, \mathrm{b}^{\prime}\right)$, the transition function between the corresponding affine charts is

$$
\phi_{\mathrm{BB}^{\prime}}:=\phi_{\mathrm{B}} \circ \phi_{\mathrm{B}^{\prime}}^{-1}: \mathbb{R}^{n} \rightarrow \mathbb{R}^{n},
$$

whose a-th component is

$$
\phi_{\mathrm{BB}^{\prime}}^{\mathrm{a}}\left(x^{1}, \cdots, x^{\mathfrak{n}}\right)=A_{b}^{\mathrm{a}} x^{\mathrm{b}}+\mathrm{a}^{\mathrm{a}},
$$

where $a^{a}:=\theta^{a}\left(o^{\prime}-o\right)$ and $A_{b}^{a}=\theta^{a}\left(e_{b}^{\prime}\right)$, i.e. $e_{b}=A_{b}^{a} e_{b}$.

\section{A.4. Passive versus active transformations}

In the physics literature these transition function between different affine charts are sometimes referred to as "passive transformations". In contrast, an "active transformation" then refers to an affine map of $M$ represented in terms of coordinates with respect to one and the same chart. So given an affine map $F$ and a single affine chart $\phi_{\mathrm{B}}$, we have

$$
\mathrm{F}_{\mathrm{B}}:=\phi_{\mathrm{B}} \circ \mathrm{F} \circ \phi_{\mathrm{B}}^{-1}: \mathbb{R}^{\mathrm{n}} \rightarrow \mathbb{R}^{\mathrm{n}}
$$

whose a-th component is

$$
\mathrm{F}_{\mathrm{B}}^{\mathrm{a}}\left(x^{1}, \cdots, x^{\mathfrak{n}}\right)=A_{b}^{a} x^{b}+a^{a},
$$

where $a^{a}:=\theta^{a}(F(o)-o)$ and $A_{b}^{a}:=\theta^{a}\left(f\left(e_{b}\right)\right)$, i.e. $f\left(e_{b}\right)=A_{b}^{a} e_{a}$. The analytic similarity of the right-hand sides of (73b) and (74b) should not deceive us about their different meanings. Whereas (73b) relates coordinate representatives of the same point in different affine charts, (74b) relates coordinate representatives of source and image point under an affine map in one and the same affine chart. This remark extends to general tensor fields, where one can either 
relate coordinate representatives of the same field in different affine charts, or coordinate representatives of different fields in the same affine chart. Here the "different fields" are related by the action of the affine group (or subgroup thereof) on the particular bundle in which the field is a section; compare Appendix B.3. Note that changing the chart will result in a change of the coordinate representatives of all fields, whereas we may choose to let affine transformation just act on any proper subset of fields. Also, on general manifolds, passive transformations will not form a group unless all charts have the same domain.

\section{A.5. Poincaré group as metric preserving affine group}

Finally we mention the definition of the Poincare group, which we embed into a more general setting. Assume $V$ to be endowed with a "metric" $g \in V^{*} \otimes V^{*}$, that is, a non-degenerate symmetric bilinear form of any signature. This defines the generalised orthogonal subgroup,

$$
\mathrm{O}(\mathrm{V}, \mathrm{g}):=\{\mathrm{A} \in \mathrm{GL}(\mathrm{V}): \mathrm{g}(\mathrm{A} v, \mathrm{~A} w)=\mathrm{g}(v, w) \forall v, w \in \mathrm{V}\}
$$

The inhomogeneous generalised orthogonal group, which we denote by $\operatorname{IO}(M, V,+, g)$, is then defined as the preimage in $\operatorname{Aff}(M, V,+)$ of $\mathrm{O}(\mathrm{V}, \mathrm{g}) \subset \mathrm{GL}(\mathrm{V})$ under the homomorphism (70):

$$
\mathrm{IO}(\mathrm{M}, \mathrm{V},+, \mathrm{g}):=\pi^{-1}(\mathrm{O}(\mathrm{V}, \mathrm{g})) \subset \operatorname{Aff}(\mathrm{M}, \mathrm{V},+) .
$$

$\operatorname{IO}(M, V,+, g)$ is the automorphism group of the structure $(M, V,+, g)$ that comprises the affine structure $(M, V,+)$ with the metric structure $g$ on $V . \operatorname{IO}(M, V,+, g)$ is (not naturally) isomorphic to $\mathrm{V} \rtimes \mathrm{O}(\mathrm{V}, \mathrm{g})$, as is immediately seen by restricting any of the isomorphisms $\Phi_{\mathrm{o}}$ in (72) to $\mathrm{IO}(M, V,+, g) \subset \operatorname{Aff}(M, V,+)$. Now, if $g$ is of signature $(1,(n-1))$ [or $((n-1), 1)]$, in which case we write $\eta$ instead of $g$, then $O(V, g=\eta)$ is called the Lorentz group Lor, and $\operatorname{IO}(M, V,+, g=\eta)$ is called the Poincaré group, Poin, in $n$ dimensions. Hence Poin $:=\pi^{-1}(\mathrm{O}(\mathrm{V}, \mathrm{g}))$.

\section{B. Spacetime: General conventions and notation}

\section{B.1. The notion of spacetime}

In physics the notion of "spacetime" is usually taken to imply a tuple $(M, g)$ in which the first entry, $M$, is a $n \geq 3$-dimensional (usually $n=4$ ) differentiable manifold which is connected, Hausdorff, paracompact, and orientable. The second entry, g, represents a Lorentzian metric with respect to which $M$ is time orientable and usually (but not always) assumed to be globally hyperbolic. These latter two properties refer to the pair $(M, g)$ and are discussed in a little more detail below. Let us begin by discussing aspects of $M$ alone.

\section{B.2. Some obvious bundles over manifolds}

As usual, the tangent and cotangent spaces at a point $m \in M$ are denoted by $T_{m} M$ and $T_{m}^{*} M$, respectively and the tangent and cotangent bundles by $T M:=\bigcup_{m \in M} T_{m} M$ and $T^{*} M:=$ 
$\bigcup_{m \in M} T_{m}^{*} M$, respectively. The tensor product of the $p$-fold tensor product of $T_{m} M$ with the $q$-fold tensor product of $T_{m}^{*} M$ is called $T_{m}{ }_{q}^{p} M$. It is said to consist of $p$-fold contravaraint and $q$-fold covariant tensors at $m \in M$. The bundle of such tensors is denoted by $T_{q}^{p} M:=$ $\bigcup_{m \in M} T_{m}{ }_{q}^{p} M$. The symbols $\otimes, \vee$, and $\wedge$ denote the tensor product, the symmetrised, and antisymmetrised tensor product, respectively. We shall mainly use the antisymmetrised tensor product which is explicitly defined in (83). The symmetrised product is defined analogously just without the sign-function $\operatorname{sign}(\sigma)$ in $(79)$. We shall also make use of the inner-product structure that the antisymmetric tensor product inherits from that of its factors. Since here various conventions concerning combinatorial factors as well as signs enter, which will be important to keep in mind in order to compare formulae from different sources, we have collected all our conventions and constructions in a self-contained exposition in Appendix C. This includes in particular the Hodge duality map, that we shall denote as usual by $\star$. The $p$-fold antisymmetric tensor product of $\mathrm{T}_{\mathrm{m}}^{*} M$ is denoted by $\wedge^{\mathrm{p}} \mathrm{T}_{\mathrm{m}}^{*} M$ and the bundle of such forms accordingly by $\wedge^{\mathrm{p}} \mathrm{T}^{*} \mathrm{M}$. A section $\Lambda \in \Gamma\left(\Lambda^{p} \mathrm{~T}^{*} \mathrm{M}\right)$ is called closed if its exterior differential vanishes: $\mathrm{d} \Lambda=0$. It is called exact if $\Lambda=\mathrm{d} \lambda$ for some $\lambda \in \Gamma\left(\bigwedge^{\mathrm{p}-1} \mathrm{~T}^{*} M\right)$

\section{B.3. Natural bundles}

If $B$ denotes any bundle over $M$, including all the ones mentioned so far, we denote by $\Gamma(B)$ the linear space of all its smooth (say $C^{\infty}$ ) sections. Dynamical laws in physics are differential equations that elements of $\Gamma(\mathrm{B})$ have to satisfy. In this paper we are interested in symmetries of such equations, that is, operations on $\Gamma(\mathrm{B})$ under which the subset of solutions stays invariant (as set). Of particular interest for us are symmetries that derive from symmetries of spacetime $(M, g)$, e.g., an action $\Phi$ of a finite-dimensional Lie group $G$ on $M$ in the sense of (43). In order to meaningfully ask whether this action defines a symmetry of a differential equation on $\Gamma(\mathrm{B})$ we must first know how the action $\Phi$ lifts to an action $\hat{\Phi}$ on $\Gamma(\mathrm{B})$, i.e. a homomorphism $\hat{\Phi}: G \rightarrow \operatorname{Diff}(B)$ such that for all $g \in G$ we have $\pi \circ \widehat{\Phi}_{g}=\Phi_{g} \circ \pi$, where $\pi: B \rightarrow M$ denotes the bundle projection. Now, a bundle B is called natural if such a lift exists naturally for all $\Phi \in \operatorname{Diff}(M)$ [15]. This is, e.g., the case for all bundles discussed above: Just take the push-forward of $\Phi$ on vector fields, the pull-back of $\Phi^{-1}$ on form fields, and extend naturally to tensor products of those. Generally it is true that $\Phi$ naturally lifts from $M$ to the principal bundle $B=F L(M)$ of linear frames (by push-forward of each frame) and hence to all bundles associated to $\mathrm{FL}(M)$. In particular this implies that the Lie derivative is defined for all sections in natural bundles. On the other hand, there exist physically relevant bundles which are not natural in that sense, like, e.g., the principal bundle of orthogonal (with respect to some metric $\mathrm{g}$ ) frames $\mathrm{FO}(M, g)$ and its associated bundles, and also the double cover $\overline{\mathrm{FO}}(\mathrm{M}, \mathrm{g})$, an important associated bundle of which is the bundles of spinors. In these cases natural lifts exist only for the subgroup of isometries $(M, g)$ within $\operatorname{Diff}(M)$. This implies that the ordinary Lie derivative of spinor fields exists only with respect to vector fields generating isometries (Killing fields). For our purpose, in particular in connection with the discussion of Section 2.3, this is sufficient. But we mention that there exist generalisations of the ordinary notion of a Lie derivative that do apply to spinor fields such that derivatives with respect to all vector fields make sense. Compare, e.g., the construction in [3] and the more systematic treatment in [6] within the framework of 
gauge theories.

\section{B.4. Lorentzian manifolds}

As already anticipated above, we shall endow $M$ with a Lorentzian metric $g \in \Gamma\left(T^{*} M V\right.$ $\left.T^{*} M\right)$. That means that $g(m) \in T_{m}^{*} M \otimes T_{m}^{*} M$ is a non-degenerate symmetric bilinear form of signature $(1,-1, \cdots,-1)$ at each piont $m$. There is a slight conventional ambiguity here in that sometimes the opposite signature $(-1,1, \cdots, 1)$ is taken, which for obvious reasons is referred to as the "mostly plus" convention. In this paper we stick to the "mostly minus" convention. We assume $(M, g)$ to be globally hyperbolic, which implies that $M$ is diffeomorphic to $\mathbb{R} \times \Sigma$, where $\Sigma$ is a connected $(n-1)$-dimensional Riemannian manifold with Riemannian structure $h=-\left.g\right|_{T \Sigma}$. We also assume $(M, g)$ to be time-orientable, which means that there exists a vector field $X \in \Gamma(T M)$ which is timelike (i.e. $g(X, X)>0$; hence, in particular, nowhere vanishing). At each $m \in M$ the vector $X_{m} \in T_{m} M$ selects one of the two components of the so-called "chronological cone" $\mathcal{C}_{\mathrm{m}}:=\left\{X \in \mathrm{T}_{\mathrm{m}} M: g(X, X)>0\right\} \subset \mathrm{T}_{\mathrm{m}} M$, which one may call the future component.

An orientation for $M$ is picked by specifying a volume form $\varepsilon \in \Gamma\left(\bigwedge^{n} T^{*} M\right)$. Relative to that choice, a basis $\left\{e_{0}(m), \cdots,, e_{n-1}(m)\right\}$ of $T_{m} M$ is then defined to be positively oriented, if and only if $\varepsilon_{m}\left(e_{0}(m), \cdots, e_{n-1}(m)\right)>0$. Let $\left\{e_{\alpha} \in \Gamma(T M): \alpha=0, \cdots, n-1\right\}$ be a basis at each point (such global bases exist, due to $M=\mathbb{R} \times \Sigma$, if $\Sigma$ is parallelizable, which is automatic in $n=4$ dimensions, i.e. if $\Sigma$ is 3-dimensional) and $\left\{\theta^{\alpha} \in \Gamma(T M): \alpha=0, \cdots, n-1\right\}$ be its dual basis, i.e. $\theta^{\alpha}\left(e_{\beta}\right)=\delta_{\beta}^{\alpha}$. Then we call this dual pair of bases adapted if they are orthonormal in the sense of $g\left(e_{\alpha}, e_{\beta}\right)=\eta_{\alpha, \beta}=\operatorname{diag}(1,-1, \cdots,-1$,$) , positively oriented in$ the sense of $\varepsilon\left(e_{0}, \cdots, e_{n-1}\right)>0$, and time oriented in the sense of $g\left(X, e_{0}\right)>0$. The volume form $\varepsilon$ may then be identified with the unique element in $\Gamma\left(\bigwedge^{n} T^{*} M\right)$ which assigns the value 1 (unit volume) to any adapted basis: $\varepsilon\left(e_{0}, \cdots, e_{n-1}\right)=1$; hence

$$
\varepsilon=\theta^{0} \wedge \cdots \wedge \theta^{n-1} \text {. }
$$

\section{Definitions and convention in multilinear algebra}

In this appendix we collect our definitions and conventions in a self-contained way, so as to ease comparison with the main text and also provide some explanatory material.

\section{C.1. Vector spaces and the exterior algebra of its dual space}

Let $\mathrm{V}$ be a real $n$-dimensional vector space, $\mathrm{V}^{*}$ its dual space and $\mathrm{T}^{\mathrm{p}} \mathrm{V}^{*}=\mathrm{V}^{*} \otimes \cdots \otimes \mathrm{V}^{*}$ its $\mathrm{p}$ fold tensor product. ${ }^{12} \mathrm{~T}^{\mathrm{p}} \mathrm{V}^{*}$ carries a representation $\pi_{\mathrm{p}}$ of $S_{\mathrm{p}}$, the symmetric group (permutation

\footnotetext{
${ }^{12} \mathrm{We}$ follow standard tradition to define forms, i.e. the antisymmetric tensor product on the dual vector space $\mathrm{V}^{*}$ rather than on V. Clearly, all constructions that are to follow could likewise be made in terms if Vrather than $\mathrm{V}^{*}$.
} 
group) of $p$ objects, given by

$$
\pi_{\mathrm{p}}: S_{\mathrm{p}} \rightarrow \operatorname{End}\left(\mathrm{T}^{\mathrm{p}} \mathrm{V}^{*}\right), \quad \pi_{\mathrm{p}}(\sigma)\left(\alpha_{1} \otimes \cdots \otimes \alpha_{\mathrm{p}}\right):=\alpha_{\sigma(1)} \otimes \cdots \otimes \alpha_{\sigma(\mathrm{p})}
$$

and linear extension to sums of tensor products. On $T^{p} V^{*}$ we define the linear operator of antisymmetrisation by

$$
\operatorname{Alt}_{\mathrm{p}}:=\frac{1}{\mathrm{p} !} \sum_{\sigma \in \mathrm{S}_{\mathrm{p}}} \operatorname{sign}(\sigma) \pi_{\mathrm{p}},
$$

where sign : $S_{p} \rightarrow\{1,-1\} \cong \mathbb{Z}_{2}$ is the sign-homomorphism. This linear operator is idempotent (i.e. a projection operator) and its image of $\mathrm{T}^{p} \mathrm{~V}^{*}$ under $\mathrm{Alt}_{\mathrm{p}}$ is the subspace of totally antisymmetric tensor-products. We write

$$
\pi_{\mathrm{p}}\left(\mathrm{T}^{\mathrm{p}} \mathrm{V}^{*}\right)=: \bigwedge^{\mathrm{p}} \mathrm{V}^{*}
$$

Clearly

We set

$$
\operatorname{dim}\left(\bigwedge^{p} V^{*}\right)= \begin{cases}\left(\begin{array}{l}
n \\
p
\end{array}\right) & \text { for } p \leq n \\
0 & \text { for } p>n .\end{cases}
$$

$$
\wedge \mathrm{V}^{*}:=\bigoplus_{\mathrm{p}=0}^{\mathrm{n}} \wedge^{\mathrm{p}} \mathrm{V}^{*}
$$

Let $\alpha \in \Lambda^{p} V^{*}$ and $\beta \in \Lambda^{q} V^{*}$, then we define their antisymmetric tensor product

$$
\alpha \wedge \beta:=\frac{(p+q) !}{p ! q !} \operatorname{Alt}_{p+q}(\alpha \otimes \beta) \in \bigwedge^{p+q} V^{*} .
$$

One easily sees that

$$
\alpha \wedge \beta=(-1)^{p q} \beta \wedge \alpha .
$$

Bilinear extension of $\Lambda$ to all of $\Lambda V^{*}$ endows it with the structure of a real $2^{n}$-dimensional associative algebra, the so-called exterior algebra over $\mathrm{V}^{*}$. If $\alpha_{1}, \cdots, \alpha_{p}$ are in $\mathrm{V}^{*}$, we have

$$
\alpha_{1} \wedge \cdots \wedge \alpha_{p}=\sum_{\sigma \in S_{p}} \operatorname{sign}(\sigma) \alpha_{\sigma(1)} \otimes \cdots \otimes \alpha_{\sigma(p)},
$$

as one easily shows from (83) and (84) using induction.

If $\left\{\theta^{1}, \cdots, \theta^{\mathfrak{n}}\right\}$ is a basis of $V^{*}$, a basis of $\Lambda^{p} V^{*}$ is given by the following $\left(\begin{array}{l}\mathfrak{n} \\ p\end{array}\right)$ vectors

$$
\left\{\theta^{a_{1}} \wedge \cdots \wedge \theta^{a_{p}} \mid 1 \leq a_{1}<a_{2}<\cdots<a_{\mathfrak{p}} \leq n\right\} .
$$

An expansion of $\alpha \in \Lambda^{p} V^{*}$ in this basis is written as follows

$$
\alpha=: \frac{1}{p !} \alpha_{a_{1} \cdots a_{p}} \theta^{a_{1}} \wedge \cdots \wedge \theta^{a_{p}}
$$

using standard summation convention and where the coefficients $\alpha_{a_{1} \cdots a_{p}}$ are totally antisymmetric in all indices. On the level of coefficients, (83) reads

$$
(\alpha \wedge \beta)_{a_{1} \cdots a_{p+q}}=\frac{(p+q) !}{p ! q !} \alpha_{\left[a_{1} \cdots a_{p}\right.} \beta_{\left.a_{p+1} \cdots a_{p+q}\right]},
$$

where square brackets denote total antisymmetrisation in all indices enclosed:

$$
\alpha_{\left[a_{1} \cdots a_{\mathfrak{p}}\right]}:=\frac{1}{p !} \sum_{\sigma \in S_{p}} \operatorname{sign}(\sigma) \alpha_{a_{\sigma(1)} \cdots a_{\sigma(p)}} .
$$




\section{C.2. Inner products on vector spaces and their extension to the dual space and its exterior algebra}

Suppose there is an inner product (non-degenerate symmetric bilinear form) $g$ on $V$, i.e., $g$ : $\mathrm{V} \times \mathrm{V} \rightarrow \mathbb{R} ;(v, w) \mapsto \mathrm{g}(v, w)$. We do not need to restrict to any specific signature of $\mathrm{g}$ which we therefore leave open. If the signature is Lorentzian we shall use the letter $\eta$ instead of $g$, as sometimes done in the main text. A non generate $G$ defines an isomorphism $g_{\downarrow}: \mathrm{V} \rightarrow \mathrm{V}^{*}$ though $g_{\downarrow}(v):=g(v, \cdot)$. The inverse map is called $g_{\uparrow}$, i.e., $g_{\uparrow}:=\left(g_{\downarrow}\right)^{-1}: V^{*} \rightarrow V$. Hence the inner product $g$ on $V$ defines in a natural fashion an inner product on the dual space $V^{*}$, which we call $\mathrm{g}^{-1}: \mathrm{V}^{*} \times \mathrm{V}^{*} \rightarrow \mathbb{R} ;(\alpha, \beta) \mapsto \mathrm{g}^{-1}(\alpha, \beta)$. It is defined in the obvious way by first mapping $\alpha$ and $\beta$ into $V$ using $g_{\uparrow}$, and then taking the inner product using $\mathrm{g}$. Hence $g^{-1}:=g \circ\left(g_{\uparrow} \times g_{\uparrow}\right)$, that is, $g^{-1}(\alpha, \beta)=g\left(g_{\uparrow}(\alpha), g_{\uparrow}(\beta)\right)$. From that it immediately follows that $g_{\uparrow}(\beta)=g^{-1}(\cdot, \beta)$, if we identify $V^{* *} \cong V$ by the natural isomorphism $i: V \rightarrow V^{* *}$; $\mathfrak{i}(v)(\alpha):=\alpha(v)$. The maps $g_{\uparrow}$ and $g_{\downarrow}$ are often called the "index-raising" and "index lowering" map, respectively, or simply the "musical isomorphisms". The reason for these names is as follows: Let $\left\{e_{1}, \cdots, e_{\mathfrak{n}}\right\}\left\{\theta^{1}, \cdots, \theta^{\mathfrak{n}}\right\}$ be dual pairs of bases for $\mathrm{V}$ and $\mathrm{V}^{*}$ respectively, and $g\left(e_{a}, e_{b}\right)=: g_{a b}, g^{-1}\left(\theta^{a}, \theta^{b}\right)=: g^{a b}$. Then $g_{\downarrow}\left(v^{b} e_{b}\right)=v_{a} \theta^{a}$ and $g_{\uparrow}\left(\alpha_{b} \theta^{b}\right)=\alpha^{a} e_{a}$ with $v_{a}:=v^{b} g_{b a}$ and $\alpha^{a}:=g^{a b} \alpha_{b}$; also $g^{a c} g_{b c}=g^{c a} g_{c b}=\delta_{b}^{a}$, which justifies the notation $g^{-1}$, since for symmetric $g$ the matrix $\left\{g^{a b}\right\}$ is the inverse of the matrix $\left\{g_{a b}\right\}$ (for non-symmetric $g$ it is the transposed inverse). Even though not relevant here, we remark that all relations in this paragraph are deliberately written in a way that remains valid for non-symmetric $g$. To end this paragraph we also remark that if the metric with respect to which index lowering and raising is clear from the context, one often writes (so-calles "musical isomorphisms")

$$
\begin{aligned}
v^{b} & :=g_{\downarrow}(v)=g(v, \cdot), \\
\alpha^{\sharp} & :=g_{\uparrow}(\alpha)=g^{-1}(\cdot, \alpha) .
\end{aligned}
$$

We make use of the notation (90a) throughout the main text.

The inner product $\mathrm{g}^{-1}$ on $\mathrm{V}^{*}$ extends to an inner product on each $\mathrm{T}^{\mathrm{p}} \mathrm{V}^{*}$ by

$$
\left\langle\alpha_{1} \otimes \cdots \otimes \alpha_{p}, \beta_{1} \otimes \cdots \otimes \beta_{p}\right\rangle:=\prod_{a=1}^{p} g^{-1}\left(\alpha_{a}, \beta_{a}\right)
$$

and bilinear extension:

$$
\left\langle\alpha_{a_{1} \cdots a_{p}} \theta^{a_{1}} \otimes \cdots \otimes \theta^{a_{p}}, \beta_{b_{1} \cdots b_{p}} \theta^{b_{1}} \otimes \cdots \otimes \theta^{b_{p}}\right\rangle=\alpha_{a_{1} \cdots a_{p}} \beta^{a_{1} \cdots a_{p}} .
$$

In particular, it extends to each subspace $\bigwedge^{p} V^{*} \subset T^{p} V^{*}$. We have

$$
\left\langle\alpha_{1} \wedge \cdots \wedge \alpha_{p}, \beta_{1} \wedge \cdots \wedge \beta_{p}\right\rangle:=p ! \sum_{\sigma \in S_{p}} \operatorname{sign}(\sigma) \prod_{a=1}^{p} g^{-1}\left(\alpha_{a}, \beta_{\sigma(a)}\right)
$$

and hence

$$
\left\langle\frac{1}{p !} \alpha_{a_{1} \cdots a_{p}} \theta^{a_{1}} \wedge \cdots \wedge \theta^{a_{p}}, \frac{1}{p !} \beta_{b_{1} \cdots b_{p}} \theta^{b_{1}} \wedge \cdots \wedge \theta^{b_{p}}\right\rangle=\alpha_{a_{1} \cdots a_{p}} \beta^{a_{1} \cdots a_{p}} .
$$


In the totally antisymmetric case it is more convenient to renormalise this product in a $p$ dependent fashion. One sets

$$
\langle\cdot, \cdot\rangle_{\text {norm }}\left|\bigwedge^{p} V^{*}:=\frac{1}{p !}\langle\cdot, \cdot\rangle\right| \bigwedge^{p} V^{*}
$$

so that

$$
\left\langle\frac{1}{p !} \alpha_{a_{1} \cdots a_{p}} \theta^{a_{1}} \wedge \cdots \wedge \theta^{a_{p}}, \frac{1}{p !} \beta_{b_{1} \cdots b_{p}} \theta^{b_{1}} \wedge \cdots \wedge \theta^{b_{p}}\right\rangle_{n o r m}=\frac{1}{p !} \alpha_{a_{1} \cdots a_{p}} \beta^{a_{1} \cdots a_{p}} .
$$

\section{C.3. Hodge duality}

Given a choice $\mathrm{o}$ of an orientation of $\mathrm{V}^{*}$ (e.g. induced by an orientation of $\mathrm{V}$ ), there is a unique top-form $\varepsilon \in \Lambda^{n} \mathrm{~V}^{*}$ (i.e. a volume form for $\mathrm{V}$ ), associated with the triple $\left(\mathrm{V}^{*}, \mathrm{~g}^{-1}, \mathrm{o}\right)$, given by

$$
\varepsilon:=\theta^{1} \wedge \cdots \wedge \theta^{n},
$$

where $\left\{\theta^{1}, \cdots, \theta^{n}\right\}$ is any $\mathrm{g}^{-1}$-orthonormal Basis of $\mathrm{V}^{*}$ in the orientation class o. The Hodge duality map at level $0 \leq \mathrm{p} \leq \mathrm{n}$ is a linear isomorphism

$$
\star_{p}: \bigwedge^{p} V^{*} \rightarrow \bigwedge^{n-p} V^{*}
$$

defined implicitly by

$$
\alpha \wedge \star_{p} \beta=\varepsilon\langle\alpha, \beta\rangle_{\text {norm }} .
$$

This means that the image of $\beta \in \Lambda^{p} V^{*}$ under $\star_{p}$ in $\Lambda^{n-p} V^{*}$ is defined by the requirement that (98b) holds true for all $\alpha \in \Lambda^{p} V^{*}$. Linearity is immediate and uniqueness of $\star_{p}$ follows from the fact that if $\lambda \in \Lambda^{n-p} V^{*}$ and $\alpha \wedge \lambda=0$ for all $\alpha \in \Lambda^{p} V^{*}$, then $\lambda=0$. To show existence it is sufficient to define $\star_{p}$ on basis vectors. Since (98b) is also linear in $\alpha$ it is sufficient to verify (98b) if $\alpha$ runs through all basis vectors.

From now on we shall follow standard practice and drop the subscript $p$ on $\star$, supposing that this will not cause confusion.

Let $\left\{e_{1}, \cdots e_{n}\right\}$ be a basis of $V$ and $\left\{\theta^{1}, \cdots, \theta^{n}\right\}$ its dual basis of $V^{*}$; i.e. $\theta^{a}\left(e_{b}\right)=\delta_{b}^{a}$. Let further $\left\{\theta_{1}, \cdots, \theta_{\mathfrak{n}}\right\}$ be the basis of $\mathrm{V}^{*}$ given by the image of $\left\{e_{1}, \cdots e_{\mathfrak{n}}\right\}$ under $g_{\downarrow}$, i.e. $\theta_{a}=g_{a b} \theta^{b}$. Then, on the basis $\left\{\theta_{a_{1}} \wedge \cdots \wedge \theta_{a_{p}} \mid 1 \leq a_{1}<a_{2}<\cdots<a_{p} \leq n\right\}$ of $\wedge^{p} V^{*}$ the map $\star$ has the simple form

$$
\star\left(\theta_{b_{1}} \wedge \cdots \wedge \theta_{b_{p}}\right)=\frac{1}{(n-p) !} \varepsilon_{b_{1} \cdots b_{p} a_{p+1} \cdots a_{n}} \theta^{a_{p}+1} \wedge \cdots \wedge \theta^{a_{n}} .
$$

This is proven by merely checking (98b) for $\alpha=\theta^{a_{1}} \wedge \cdots \wedge \theta^{a_{p}}$ and $\beta=\theta_{b_{1}} \wedge \cdots \wedge \theta_{b_{p}}$. Instead of (99) we can write

$$
\begin{aligned}
\star\left(\theta^{a_{1}} \wedge \cdots \wedge \theta^{a_{p}}\right) & =\frac{1}{(n-p) !} g^{a_{1} b_{1}} \cdots g^{a_{p} b_{p}} \varepsilon_{b_{1} \cdots b_{p} b_{p+1} \cdots b_{n}} \theta^{b_{p+1}} \wedge \cdots \wedge \theta^{b_{n}} \\
& =\frac{1}{(n-p) !} \varepsilon^{a_{1} \cdots a_{p}}{ }_{a_{p+1} \cdots a_{n}} \theta^{a_{p}+1} \wedge \cdots \wedge \theta^{a_{n}}
\end{aligned}
$$

which makes explicit the dependence on $\varepsilon$ and $g$. 
If $\alpha=\frac{1}{p !} \alpha_{a_{1} \cdots a_{p}} \theta^{a_{1}} \wedge \cdots \wedge \theta^{a_{p}}$, then $\star \alpha=\frac{1}{(n-p) !}(\star \alpha)_{b_{1} \cdots b_{n-p}} \theta^{b_{1}} \wedge \cdots \wedge \theta^{b_{n-p}}$, where

$$
(\star \alpha)_{b_{1} \cdots b_{n-p}}=\frac{1}{p !} \alpha_{a_{1} \cdots a_{p}} \varepsilon^{a_{1} \cdots a_{p}}{ }_{b_{1} \cdots b_{n-p}} .
$$

This gives the familiar expression of Hodge duality in component language. Note that on component level the first (rather than last) $p$ indices are contracted.

Applying $\star$ twice (i.e. actually $\star_{(n-p)} \circ \star_{p}$ ) leads to the following self-map of $\Lambda^{p} V^{*}$ :

$$
\begin{aligned}
\star & \star \\
& \left.\star\left(\theta^{a_{1}} \wedge \cdots \wedge \theta^{a_{p}}\right)\right) \\
& =\frac{1}{p !(n-p) !} \varepsilon^{a_{1} \cdots a_{p}}{ }_{a_{p+1} \cdots a_{n}} \varepsilon^{a_{p+1} \cdots a_{n}}{ }_{b_{1} \cdots b_{p}} \theta^{b_{1}} \wedge \cdots \wedge \theta^{b_{p}} \\
& =\frac{(-1)^{p(n-p)}}{p !(n-p) !} \varepsilon^{a_{1} \cdots a_{p} a_{p+1} \cdots a_{n}} \varepsilon_{b_{1} \cdots b_{p} a_{p+1} \cdots a_{n}} \theta^{b_{1}} \wedge \cdots \wedge \theta^{b_{p}} \\
& =(-1)^{p(n-p)}\langle\varepsilon, \varepsilon\rangle_{\text {norm }} \theta^{a_{1}} \wedge \cdots \wedge \theta^{a_{p}} .
\end{aligned}
$$

Note that

$$
\langle\varepsilon, \varepsilon\rangle_{\text {norm }}=\frac{1}{n !} g^{a_{1} b_{1}} \cdots g^{a_{n} b_{n}} \varepsilon_{a_{1} \cdots a_{n}} \varepsilon_{b_{1} \cdots b_{n}}=\left(\varepsilon_{12 \cdots n}\right)^{2} / \operatorname{det}\left\{g\left(e_{a}, e_{b}\right)\right\} .
$$

This formula holds for any volume form $\varepsilon$ in the definition (98b), independent of whether or not it is related to $\mathrm{g}$.

Since the right-hand side of (98b) is symmetric under the exchange $\alpha \leftrightarrow \beta$, so must be the left-hand side. Using (102) we get

$$
\begin{aligned}
\langle\alpha, \beta\rangle_{\text {norm }} \varepsilon & =\alpha \wedge \star \beta=\beta \wedge \star \alpha=(-1)^{p(n-p)} \star \alpha \wedge \beta \\
& =\langle\varepsilon, \varepsilon\rangle_{\text {norm }}^{-1} \star \alpha \wedge \star \star \beta=\langle\varepsilon, \varepsilon\rangle_{\text {norm }}^{-1}\langle\star \alpha, \star \beta\rangle_{\text {norm }} \varepsilon
\end{aligned}
$$

hence

$$
\langle\star \alpha, \star \beta\rangle_{\text {norm }}=\langle\varepsilon, \varepsilon\rangle_{\text {norm }}\langle\alpha, \beta\rangle_{\text {norm }} .
$$

From this and (102) it follows for $\alpha \in \Lambda^{p} V^{*}$ and $\beta \in \Lambda^{n-p} V^{*}$, that

$$
\langle\alpha, \star \beta\rangle_{\text {norm }}=\langle\varepsilon, \varepsilon\rangle_{\text {norm }}^{-1}\langle\star \alpha, \star \star \beta\rangle_{\text {norm }}=(-1)^{p(n-p)}\langle\star \alpha, \beta\rangle_{\text {norm }} .
$$

This shows that the adjoint map of $\star$ relative to $\langle\cdot, \cdot\rangle_{\text {norm }}$ is $(-1)^{p(n-p)} \star$.

Formulae (102), (104)(105), and (106) are valid for general $\varepsilon$ in the definition (98b). If we choose $\varepsilon$ as the unique volume form that assigns unit volume to an oriented orthonormal frame, as does (97), then we have

$$
\langle\varepsilon, \varepsilon\rangle_{\text {norm }}=(-1)^{n_{-}}
$$

where $n_{-}$is the maximal dimension of subspaces in $V$ restricted to which $g$ is negative definite; i.e. $g$ is of signature $\left(n_{+}, n_{-}\right)$. Equation (105) then shows that $\star$ is an isometry for even $n_{-}$ and an anti-isometry for odd $n_{-}$. If $g$ is a Lorentzian inner product in $n$ dimensions then either $n_{+}=(n-1)$ and $n_{-}=1$, which is called the "mostly-plus convention", or $n_{+}=1$ and $n_{-}=$ $(n-1)$, which accordingly is called the "mostly-minus convention". If $n$ is even $n_{-}$is odd in both conventions, and no differences in signs occur. In contrast, for odd space-time dimensions 
all signs involving $(-1)_{-}^{n}$ differ in these two conventions. In the mostly-minus convention that we followed in the main text, we have then $\star_{n-p} \circ \star_{p}=(-1)^{(n+1)(p+1)} i d \mid \wedge^{p} V^{*}$, where we used the following identity for integers $\bmod (2): p(n-p)+(n-1)=n p+p+n+1=(n+1)(p+1)$. This means that in even space-time dimensions (like the $n=4$ we are mostly interested in) $\star$ squares to the identity on odd forms and to minus the identity on even forms, whereas in odd space-time dimensions it always squares to the identity. As explained above, this remains true for even $n$ in the mostly-plus convention, but turns to the opposite signs for odd $n$ if the conventions are changed.

Finally we note the following useful formula: If $v \in V$ let $i_{v}: T^{p} V^{*} \rightarrow T^{p-1} V^{*}$ the map which inserts $v$ into the first tensor factor. It restricts to a map $i_{v}: \Lambda^{p} V^{*} \rightarrow \Lambda^{p-1} V^{*}$. Then, for any $\alpha \in \Lambda^{\mathfrak{p}} \mathrm{V}^{*}$, we have

$$
i_{v} \star \alpha=\star\left(\alpha \wedge v^{b}\right) .
$$

using the notation (90a). It suffices to prove this for basis elements $v=e_{a}$ of $\mathrm{V}$ and $\alpha=$ $\theta^{a_{1}} \wedge \ldots \wedge \theta^{a_{p}}$ of $\wedge^{p} V^{*}$, which is almost immediate using (100).

An immediate consequence of (108) is that for any $v \in V$ we have the identity $i_{v} \star v^{b}=$ $\star\left(v^{b} \wedge v^{b}\right)=0$ and hence that $i_{v}\left(v^{b} \wedge \star v^{b}\right)=g(v, v) \star v^{b}$. On the other hand, from (98b) the left hand side of the last equation also equals to $\left\langle v^{b}, v^{b}\right\rangle i_{v} \varepsilon$. Since $g(v, v)=\left\langle v^{b}, v^{b}\right\rangle_{\text {norm }}$ we have

$$
\star v^{b}=i_{v} \varepsilon .
$$




\section{References}

[1] Ralph Abraham and Jerrold E. Marsden. Foundations of Mechanics. The Benjamin/Cummings Publishing Company, Reading, Massachusetts, second edition, 1978.

[2] Robert Beig and Niall Ó Murchadha. The Poincaré group as symmetry group of canonical general relativity. Annals of Physics, 174:463-498, 1987.

[3] Jean-Pierre Bourguignon and Paul Gauduchon. Spineurs, opérateurs de Dirac et variations de métriques. Communications in Mathematical Physics, 144(3):581-599, 1992.

[4] Steve Carlip. Kinetic energy and the equivalence principle. American Journal of Physics, 66(5):409-413, 1998.

[5] Jürgen Ehlers and Egon Köhler. Path structures on manifolds. Journal of Mathematical Physics, 18(10):2014-2018, 1977.

[6] Lorenzo Fatibene and Mauro Francaviglia. Natural and Gauge Natural Formalism for Classical Field Theory. Springer-Science+Business Media, B.V., Dordrecht, 2003.

[7] Arthur E. Fischer and Jerrold E. Marsden. Topics in the dynamics of general relativity. In Jürgen Ehlers, editor, Isolated Gravitating Systems in General Relativity, volume LXVII of Proceedings of the International School of Physics "Enrico Fermi", pages 322-395. North-Holland Publ. Comp., Amsterdam, 1979.

[8] Vladimir Fock. The Theory of Space Time and Gravitation. Pergamon Press, Oxford, second revised english edition, 1964.

[9] Hans Freudenthal. Über die Enden topologischer Räume und Gruppen. Mathematische Annalen, 33(1):692-713, 1931.

[10] Domenico Giulini. Das Problem der Trägheit. Philosophia Naturalis, 39(2):843-374, 2002.

[11] Domenico Giulini. Dynamical and Hamiltonian formulation of General Relativity. In Abhay Ashtekar and Vesselin Petkov, editors, Springer Handbook of Spacetime, pages 323-362. Springer Verlag, Berlin, 2014. Extended version (76 pages) at arXiv:1505.01403.

[12] Domenico Giulini. Energy-momentum tensors and motion in special relativity. In D. Pützfeld, C. Lämmerzahl, and B. Schutz, editors, Equations of Motion in Relativistic Gravity, volume 179 of Fundamental Theories of Physics. Springer Verlag, Dordrecht, 2015. arXiv: 1502.03930.

[13] Friedrich W. Hehl and Yuri N. Obukhov. Foundations of Classical Electrodynamics: Charge, Flux, and Metric, volume 33 of Progress in Mathematical Physics. Birkhäuser Verlag, Boston, 2003.

[14] Felix Klein. Über die Integralform der Erhaltungsgesetze und die Theorie der räumlichgeschlossenen Welt. Nachrichten von der Königlichen Gesellschaft der Wissenschaften zu Göttingen, mathematisch-physikalische Klasse, pages 394-423, 1918.

[15] Ivan Kolář, Peter W. Michor, and Jan Slovák. Natural Operations in Differential Ge- 
ometry. Springer Verlag, Berlin, 1993. Second corrected version available online: www.mat.univie.ac.at/ michor/kmsbookh.pdf.

[16] Lev Davidovich Landau and Evgeny Mikhailovich Lifshitz. The Classical Theory of Fields, volume 2 of Course of Theoretical Physics. Butterworth-Heinemann, Amsterdam, fourth revised english edition, 1980.

[17] Max Laue. Zur Dynamik der Relativitätstheorie. Annalen der Physik, 340(8):524-542, 1911. Gesammelte Schriften und Vorträge (Friedrich Vieweg \& Sohn, Braunschweig, 1961), Vol. I, p 524-542.

[18] Max Laue. Zur Theorie des Versuchs von Trouton und Noble. Annalen der Physik, 343(7):370-384, 1912.

[19] Herbert Pfister and Markus King, editors. Inertia and Gravitation. The Fundamental Nature and Structure of Space-Time, volume 897 of Lecture Notes in Physics. Springer Verlag, Cham (Switzerland), 2015.

[20] Henri Poincaré. Sur la dynamique de l'électron. Rediconti del Circolo Matematico di Palermo, 21:129-176, 1906. Submitted July 1905, printed 14th December 1905.

[21] Fritz Rohrlich. Classical Charged Particles. Foundations of their Theory. Addison-Wesley Publishing Company, Reading, Massachusetts, 1965.

[22] Fritz Rohrlich. The electron: Development of the first elementary particle theory. In Jagdish Mehra, editor, The Physicist's Conception of Nature, chapter 16, pages 331-369. R. Reidel Publishing Company, Dordrecht, 1973.

[23] Rainer K. Sachs and Hung-Hsi Wu. General Relativity for Mathematicians. Springer Verlag, New York, 1977.

[24] Sarah E. Salvioli. On the theory of geometric objects. Journal of Differential Geometry, 7:257-278, 1972.

[25] Norbert Straumann. General Relativity. Springer Verlag, Berlin, second edition, 2013.

[26] Richard C. Tolman. On the use of the energy-momentum principle in General Relativity. Physical Review, 35(8):875-895, 1930.

[27] Andrzej Trautman. Foundations and current problems of general relativity. In Andrzej Trautman, Felix Pirani, and Hermann Bondi, editors, Lectures on General Relativity, volume 1 of Brandeis Summer Institute in Theoretical Physics, pages 1-248. Prentice-Hall, Inc., Englewood Cliffs, New Jersey, 1964.

[28] Changbiao Wang. Von Laue's theorem and its applications. Canadian Journal of Physics, 93(12):1470-1476, 2015. 